\title{
Principal Vertex Operator Representations For Toroidal Lie Algebras
}

\author{
Yuly Billig * \\ Department of Mathematics and Statistics, University of New Brunswick, \\ Fredericton, N.B., E3B 5A3, Canada
}

\section{Introduction.}

Vertex operators discovered by physicists in string theory have turned out to be important objects in mathematics. One can use vertex operators to construct various realizations of the irreducible highest weight representations for affine Kac-Moody algebras. Two of these, the principal and homogeneous realizations, are of particular interest. The principal vertex operator construction for the affine algebra $A_{1}^{(1)}$ allows one to construct soliton solutions of the Korteweg - de Vries hierarchy of partial differential equations. On the other hand, the homogeneous realization is linked to the fundamental nonlinear Schrödinger equation $[\mathrm{Kac}]$.

S.Eswara Rao and R.V. Moody in [EM] studied the homogeneous vertex operator construction for toroidal Lie algebras. The present paper is devoted to the principal realization. Here we construct the principal vertex operator representation for the toroidal Lie algebra $\hat{\mathfrak{g}}$ which is a universal central extension of $\tilde{\mathfrak{g}}=\dot{\mathfrak{g}} \otimes \mathbb{C}\left[t_{0}^{ \pm}, \ldots, t_{n}^{ \pm}\right]$, in which $\dot{\mathfrak{g}}$ is a simply-laced simple finite-dimensional Lie algebra over $\mathbb{C}$. This generalizes the principal vertex operator realization of the basic representations of affine Lie algebras constructed in $[\mathrm{KKLW}]$.

We add the Lie algebra $\mathcal{D}$ of vector fields on a torus to the toroidal Lie algebra $\hat{\mathfrak{g}}$ to form a larger algebra $\mathfrak{g}$. This is necessary in order to have a sufficiently large principal Heisenberg subalgebra. To construct a representation of $\mathfrak{g}$ we consider the standard representation of the principal Heisenberg subalgebra on the Fock space $F$ and then extend it to all of $\mathfrak{g}$ by

* Work supported by the Natural Sciences and Engineering Research Council of Canada. 
means of the vertex operators. The module $F$ is irreducible as a module over $\mathfrak{g}$, and it is reducible over $\hat{\mathfrak{g}}$. It will be interesting to see how this representation fits in the framework of the Verma modules over the toroidal Lie algebras studied in [BC].

The vertex operator representations constructed in this paper for the toroidal Lie algebras may also be useful for quantum field theories in space-time of more than two dimensions [IKUX].

In section 1 we recall the construction of the toroidal Lie algebra and in section 2 we sketch the principal vertex operator realization of the basic representations of affine Lie algebras. In section 3 we present the principal Heisenberg subalgebra of $\mathfrak{g}$ and define its action on the Fock space. We also introduce there vertex operators that will allow us to extend this action to $\mathfrak{g}$. At the end of the section we state the main theorem of the paper. The proof of the main theorem occupies section 4. In the final section we construct the analogues of the Sugawara operators.

\section{Definitions and notations.}

Let $\dot{\mathfrak{g}}$ be a simple finite-dimensional complex Lie algebra of type $A_{\ell}, D_{\ell}$ or $E_{\ell}$ (i.e., simply-laced). The Killing form $(\cdot \mid \cdot)$ is non-degenerate on the Cartan subalgebra $\dot{\mathfrak{h}}$ of $\dot{\mathfrak{g}}$ and induces the map $\nu: \dot{\mathfrak{h}} \rightarrow \dot{\mathfrak{h}}^{*}$. Let $\dot{\Delta}$ be the root system of $\dot{\mathfrak{g}}$. Since $\dot{\mathfrak{g}}$ is simply-laced then $(\alpha \mid \alpha)=2$ for all nonzero roots $\alpha \in \dot{\Delta}$. and for root elements $e_{\alpha} \in \dot{\mathfrak{g}}^{\alpha}, e_{-\alpha} \in \dot{\mathfrak{g}}^{-\alpha}$ we have $\left[e_{\alpha}, e_{-\alpha}\right]=\left(e_{\alpha} \mid e_{-\alpha}\right) \nu^{-1}(\alpha)$.

To construct a toroidal algebra let us consider a tensor product of $\dot{\mathfrak{g}}$ with the algebra of Laurent polynomials in $n+1$ variable:

$$
\tilde{\mathfrak{g}}=\dot{\mathfrak{g}} \otimes \mathbb{C}\left[t_{0}^{ \pm}, t_{1}^{ \pm}, \ldots, t_{n}^{ \pm}\right]
$$

Then we define the toroidal Lie algebra corresponding to $\dot{\mathfrak{g}}$ as the universal central extension of $\tilde{\mathfrak{g}}$. The explicit construction of this extension is known from the works [Kas], [MEY]. Let $\dot{\mathcal{K}}$ be an $(n+1)$-dimensional space with the basis $\left\{K_{0}, K_{1}, \ldots, K_{n}\right\}$. Consider a derivation

$d_{p}=t_{p} \frac{d}{d t_{p}}$ of the algebra $\mathbb{C}\left[t_{0}^{ \pm}, t_{1}^{ \pm}, \ldots, t_{n}^{ \pm}\right]$which can be naturally extended on the tensor product

$$
\tilde{\mathcal{K}}=\dot{\mathcal{K}} \otimes \mathbb{C}\left[t_{0}^{ \pm}, t_{1}^{ \pm}, \ldots, t_{n}^{ \pm}\right]
$$

The space of the universal central extension of $\tilde{\mathfrak{g}}$ is

$$
\mathcal{K}=\tilde{\mathcal{K}} / d \tilde{\mathcal{K}}
$$


where

$$
d \tilde{\mathcal{K}}=\left\{\sum_{p=0}^{n} K_{p} \otimes d_{p}(f) \mid f \in \mathbb{C}\left[t_{0}^{ \pm}, t_{1}^{ \pm}, \ldots, t_{n}^{ \pm}\right]\right\} \subset \tilde{\mathcal{K}} .
$$

We will denote the image of $K_{p} \otimes t_{0}^{r_{0}} \mathbf{t}^{\mathbf{r}}$ in $\mathcal{K}$ by $t_{0}^{r_{0}} \mathbf{t}^{\mathbf{r}} K_{p}$, where $\mathbf{r}=\left(r_{1}, \ldots r_{n}\right)$ and $\mathbf{t}^{\mathbf{r}}=t_{1}^{r_{1}} \ldots t_{n}^{r_{n}}$. Note that $\mathcal{K}$ has the defining relations

$$
r_{0} t_{0}^{r_{0}} \mathbf{t}^{\mathbf{r}} K_{0}+r_{1} t_{0}^{r_{0}} \mathbf{t}^{\mathbf{r}} K_{1}+\ldots+r_{n} t_{0}^{r_{0}} \mathbf{t}^{\mathbf{r}} K_{n}=0
$$

The toroidal algebra is the Lie algebra

$$
\hat{\mathfrak{g}}=\dot{\mathfrak{g}} \otimes \mathbb{C}\left[t_{0}^{ \pm}, t_{1}^{ \pm}, \ldots, t_{n}^{ \pm}\right] \oplus \mathcal{K}
$$

with the bracket

$$
\left[g_{1} \otimes f_{1}\left(t_{0} \ldots t_{n}\right), g_{2} \otimes f_{2}\left(t_{0} \ldots t_{n}\right)\right]=\left[g_{1}, g_{2}\right] \otimes\left(f_{1} f_{2}\right)+\left(g_{1} \mid g_{2}\right) \sum_{p=0}^{n} d_{p}\left(f_{1}\right) f_{2} K_{p}
$$

and

$$
[\hat{\mathfrak{g}}, \mathcal{K}]=0 .
$$

Finally, we shall add certain derivations to $\hat{\mathfrak{g}}$. Specifically, let $\mathcal{D}$ be the Lie algebra of derivations of $\mathbb{C}\left[t_{0}^{ \pm}, t_{1}^{ \pm}, \ldots, t_{n}^{ \pm}\right]$:

$$
\mathcal{D}=\left\{\sum_{p=0}^{n} f_{p}\left(t_{0}, \ldots, t_{n}\right) d_{p} \mid f_{0}, \ldots, f_{n} \in \mathbb{C}\left[t_{0}^{ \pm}, t_{1}^{ \pm}, \ldots, t_{n}^{ \pm}\right]\right\}
$$

It is a general fact, that a derivation acting on a Lie algebra can be lifted in a unique way to a derivation of the universal central extension of this Lie algebra [BM]. Thus the natural action of $\mathcal{D}$ on $\tilde{\mathfrak{g}}$

$$
f_{1}\left(t_{0}, \ldots, t_{n}\right) d_{p}\left(g \otimes f_{2}\left(t_{0}, \ldots, t_{n}\right)=g \otimes f_{1} d_{p}\left(f_{2}\right)\right.
$$

has a unique extension to $\hat{\mathfrak{g}}$. We shall denote the lift of $f\left(t_{0}, \ldots, t_{n}\right) d_{p}$ by $f\left(t_{0}, \ldots, t_{n}\right) D_{p}$. Its action on the subspace $\tilde{\mathfrak{g}}$ is unchanged, while the action on $\mathcal{K}$ is given by formula [EM]:

$$
f_{1} D_{a}\left(f_{2} K_{b}\right)=f_{1} D_{a}\left(f_{2}\right) K_{b}+\delta_{a b} \sum_{p=0}^{n} f_{2} D_{p}\left(f_{1}\right) K_{p}
$$

Consider a subalgebra $\mathcal{D}_{+}$in $\mathcal{D}$ :

$$
\mathcal{D}_{+}=\left\{\sum_{p=1}^{n} f_{p}\left(t_{0}, \ldots, t_{n}\right) d_{p} \mid f_{1}, \ldots, f_{n} \in \mathbb{C}\left[t_{0}^{ \pm}, t_{1}^{ \pm}, \ldots, t_{n}^{ \pm}\right]\right\} .
$$


We will be working with the algebra $\mathfrak{g}$ which is a deformation of the semidirect product of $\hat{\mathfrak{g}}$ with $\mathcal{D}_{+}$:

$$
\mathfrak{g}=\dot{\mathfrak{g}} \otimes \mathbb{C}\left[t_{0}^{ \pm}, t_{1}^{ \pm}, \ldots, t_{n}^{ \pm}\right] \oplus \mathcal{K} \oplus \mathcal{D}_{+}
$$

Here multiplication in $\hat{\mathfrak{g}}$ is given by (1.1) and (1.2), the action of $\mathcal{D}_{+}$on $\tilde{\mathfrak{g}}$ is given by (1.3) and on $\mathcal{K}$ by (1.4). The Lie bracket in $\mathcal{D}_{+}$is the following:

$$
\left[t_{0}^{r_{0}} \mathbf{t}^{\mathbf{r}} D_{a}, t_{0}^{m_{0}} \mathbf{t}^{\mathbf{m}} D_{b}\right]=m_{a} t_{0}^{r_{0}+m_{0}} \mathbf{t}^{\mathbf{r}+\mathbf{m}} D_{b}-r_{b} t_{0}^{r_{0}+m_{0}} \mathbf{t}^{\mathbf{r}+\mathbf{m}} D_{a}-m_{a} r_{b}\left\{\sum_{p=0}^{n} r_{p} t_{0}^{r_{0}+m_{0}} \mathbf{t}^{\mathbf{r}+\mathbf{m}} K_{p}\right\}
$$

The last term in this formula is the correction term to the ordinary Lie bracket in $\mathcal{D}$. This abelian deformation of the Lie algebra of vector fields on a torus is a generalization of the Virasoro algebra and was introduced in [EM].

\section{Principal realization of the basic representation of affine Lie algebra.}

When $n=0$ the algebra $\mathfrak{g}$ constructed above yields the derived affine Kac-Moody algebra. We set $n=0$ throughout this section. In this case $\mathcal{K}$ is one-dimensional and is spanned by $K_{0}$, while $\mathcal{D}_{+}$is trivial, so

$$
\mathfrak{g}=\dot{\mathfrak{g}} \otimes \mathbb{C}\left[t_{0}, t_{0}^{-1}\right] \oplus \mathbb{C} K_{0}
$$

Let us recall the principal realization of the basic representation of $\mathfrak{g}$ (see [Kac] for details). The starting point of this construction is the principal Heisenberg subalgebra in $\mathfrak{g}$.

Let $\left\{\alpha_{1}, \ldots, \alpha_{\ell}\right\}$ be simple roots and $\theta$ be the highest positive root in $\dot{\Delta}$. We define the height for a root $\beta=\sum_{i=1}^{\ell} k_{i} \alpha_{i}$ as

$$
\operatorname{ht}(\beta)=\sum_{i=1}^{\ell} k_{i}
$$

Note that $h t(\theta)=h-1$, where $h$ is a Coxeter number of $\dot{\mathfrak{g}}[\mathrm{Kos}]$.

Consider the principle gradation of $\tilde{\mathfrak{g}}$ defined by

$$
\begin{gathered}
\operatorname{deg}\left(\dot{\mathfrak{g}}^{\alpha_{1}} \otimes 1\right)=\ldots=\operatorname{deg}\left(\dot{\mathfrak{g}}^{\alpha_{\ell}} \otimes 1\right)=\operatorname{deg}\left(\dot{\mathfrak{g}}^{-\theta} \otimes t_{0}\right)=1, \\
\operatorname{deg}\left(\dot{\mathfrak{g}}^{-\alpha_{1}} \otimes 1\right)=\ldots=\operatorname{deg}\left(\dot{\mathfrak{g}}^{-\alpha_{\ell}} \otimes 1\right)=\operatorname{deg}\left(\dot{\mathfrak{g}}^{\theta} \otimes t_{0}^{-1}\right)=-1 .
\end{gathered}
$$


We choose nonzero root vectors $e_{0} \in \dot{\mathfrak{g}}^{-\theta} \otimes t_{0}, e_{1} \in \dot{\mathfrak{g}}^{\alpha_{1}} \otimes 1, \ldots, e_{\ell} \in \dot{\mathfrak{g}}^{\alpha_{\ell}}$ and form the element $\bar{e}=\sum_{i=0}^{\ell} e_{i} \in \tilde{\mathfrak{g}}$. Since $\bar{e}$ is of degree 1 then its centralizer $\tilde{\mathfrak{s}}$ in $\tilde{g}$ is homogeneous with respect to the gradation.

Next, consider a projection

$$
\pi: \dot{\mathfrak{g}} \otimes \mathbb{C}\left[t_{0}, t_{0}^{-1}\right] \rightarrow \dot{\mathfrak{g}} \quad \text { by } \quad t_{0} \mapsto 1 .
$$

Note that $\operatorname{deg}\left(\dot{\mathfrak{g}}^{-\theta} \otimes t_{0}\right)=1$ and $\operatorname{deg}\left(\dot{\mathfrak{g}}^{-\theta} \otimes 1\right)=h t(-\theta)=-h+1$, while $\pi\left(\dot{\mathfrak{g}}^{-\theta} \otimes t_{0}\right)=$

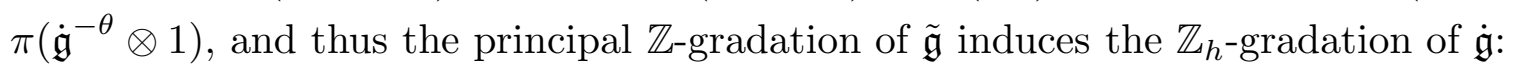

$$
\dot{\mathfrak{g}}=\sum_{j \in \mathbb{Z}_{h}} \dot{\mathfrak{g}}_{j} .
$$

The element $\pi(\bar{e})$ is regular in $\dot{\mathfrak{g}}$, hence its centralizer $\dot{\mathfrak{s}}=\pi(\tilde{\mathfrak{s}})$ in $\dot{\mathfrak{g}}$ is a Cartan subalgebra, which implies that both $\dot{\mathfrak{s}}$ and $\tilde{\mathfrak{s}}=\dot{\mathfrak{s}} \otimes \mathbb{C}\left[t_{0}, t_{0}^{-1}\right]$ are abelian.

The preimage $\mathfrak{s}=\dot{\mathfrak{s}} \otimes \mathbb{C}\left[t_{0}, t_{0}^{-1}\right] \oplus \mathbb{C} K_{0}$ of $\tilde{\mathfrak{s}}$ in $\mathfrak{g}$ is an infinite-dimensional Heisenberg algebra which is called the principal Heisenberg subalgebra of $\mathfrak{g}$. This subalgebra is the cornerstone of the construction of the vertex operator representation of $\mathfrak{g}$. The scheme of this construction is the following: one starts with the standard irreducible representation of $\mathfrak{s}$ and then magically one can lift this representation to the whole of $\mathfrak{g}$. Moreover the action of $\mathfrak{g}$ is prescribed and given by vertex operators. Finally one has only to check in one way or another that this construction works.

The irreducible representation of $\mathfrak{g}$ constructed in this way is of the highest weight $\Lambda_{0}$, where the linear functional $\Lambda_{0}$ is given (we consider the simply-laced case) by

$$
\Lambda_{0}\left(\nu^{-1}\left(\alpha_{i}\right) \otimes t_{0}^{0}\right)=0, \quad \Lambda_{0}\left(K_{0}\right)=1 .
$$

This highest weight representation is called the basic representation of $\mathfrak{g}$.

The Cartan subalgebra $\dot{\mathfrak{s}}$ is homogeneous with respect to the principle $\mathbb{Z}_{h}$-gradation:

$$
\dot{\mathfrak{s}}=\sum_{j \in \mathbb{Z}_{h}} \dot{\mathfrak{s}}_{j} .
$$

Since $\left(\dot{\mathfrak{g}}_{i} \mid \dot{\mathfrak{g}}_{j}\right)=0$ unless $i+j=0(\bmod h)$ and $(\cdot \mid \cdot)$ is nondegenerate on $\dot{\mathfrak{s}}$ then one can choose a basis in $\dot{\mathfrak{s}}:\left\{T_{1}, T_{2}, \ldots, T_{\ell}\right\}$ such that $T_{i} \in \dot{\mathfrak{g}}_{m_{i}}$, where $1 \leq m_{1} \leq m_{2} \leq \ldots \leq$ $m_{\ell}<h$ and

$$
\left(T_{i} \mid T_{\ell+1-j}\right)=h \delta_{i j} .
$$

The numbers $\left\{m_{1}, \ldots, m_{\ell}\right\}$ are called the exponents of $\dot{\mathfrak{g}}[\mathrm{Kos}]$. Note that $\dot{\mathfrak{s}} \cap \dot{\mathfrak{h}}=(0)$, and therefore zero is not an exponent. Also, $m_{\ell+1-i}=h-m_{i}$. 
The principal Heisenberg subalgebra $\mathfrak{s}$ is spanned by $T_{i} \otimes t_{0}^{j}, j \in \mathbb{Z}, i=1, \ldots, \ell$ and $K_{0}$. We can now write the multiplication in $\mathfrak{s}$ explicitly:

$$
\left[T_{i_{1}} \otimes t_{0}^{j_{1}}, T_{\ell+1-i_{2}} \otimes t_{0}^{j_{2}}\right]=h j_{1} \delta_{i_{1} i_{2}} \delta_{j_{1},-j_{2}}
$$

It turns out that it is more convenient to work with a slightly different realization of $\mathfrak{g}$ based on the $\mathbb{Z}_{h}$-gradation of $\dot{\mathfrak{g}}$. Consider

$$
\mathfrak{g}_{s}=\sum_{j \in \mathbb{Z}} \dot{\mathfrak{g}}_{j} \otimes s^{j} \oplus \mathbb{C} K_{0}
$$

with the Lie bracket

$$
\begin{gathered}
{\left[g_{1} \otimes s^{i}, g_{2} \otimes s^{j}\right]=\left[g_{1}, g_{2}\right] \otimes s^{i+j}+\frac{i}{h}\left(g_{1} \mid g_{2}\right) \delta_{i,-j} K_{0},} \\
{\left[K_{0}, \mathfrak{g}_{s}\right]=0 .}
\end{gathered}
$$

The proof of the following lemma is straightforward, so we omit it.

Lemma 1. The Lie algebras $\mathfrak{g}$ and $\mathfrak{g}_{s}$ are isomorphic and the isomorphism

$$
\psi: \dot{\mathfrak{g}} \otimes \mathbb{C}\left[t_{0}, t_{0}^{-1}\right] \oplus \mathbb{C} K_{0} \rightarrow \sum_{j \in \mathbb{Z}} \dot{\mathfrak{g}}_{j} \otimes s^{j} \oplus \mathbb{C} K_{0}
$$

is given by

$$
\begin{gathered}
\psi\left(e_{\alpha} \otimes t^{i}\right)=e_{\alpha} \otimes s^{\mathrm{ht}(\alpha)+i h} \\
\psi\left(\nu^{-1}(\alpha) \otimes t^{i}\right)=\nu^{-1}(\alpha) \otimes s^{i h}+\delta_{i, 0} \frac{\mathrm{ht}(\alpha)}{h} K_{0} \\
\psi\left(K_{0}\right)=K_{0} .
\end{gathered}
$$

We shall identify $\mathfrak{g}$ and $\mathfrak{g}_{s}$ using this isomorphism.

In order to describe a basis in the principal Heisenberg subalgebra $\psi(\mathfrak{s})$ we need to introduce the sequence $\left\{b_{i}\right\}_{i \in \mathbb{Z}}$ such that $b_{i+j \ell}=m_{i}+j h$ for $j \in \mathbb{Z}, i=1, \ldots, \ell$. Then $\psi(\mathfrak{s})$ is spanned by $\left\{T_{i} \otimes s^{b_{i}}, K_{0}\right\}, i \in \mathbb{Z}$. Note that $b_{1-i}=-b_{i}$.

Let $\dot{\Delta}_{s}$ be the root system of $\dot{\mathfrak{g}}$ with respect to the Cartan subalgebra $\dot{\mathfrak{s}}$. For $\alpha \in \dot{\Delta}_{s}$ choose a root element

$$
A^{\alpha}=\sum_{j \in \mathbb{Z}_{h}} A_{j}^{\alpha}
$$

Since $\pi(\bar{e})$ is a regular element of the Cartan subalgebra then $\left[\pi(\bar{e}), A^{\alpha}\right]=\lambda^{\alpha} A^{\alpha}$ with $\lambda^{\alpha} \neq 0$. Hence $A_{j}^{\alpha}=\left(\frac{\operatorname{ad} \pi(\bar{e})}{\lambda^{\alpha}}\right)^{j} A_{0}^{\alpha}$ and all the components $A_{j}^{\alpha}$ are nonzero. We let the 
indices $i, j$ in $T_{i}$ and $A_{j}^{\alpha}$ run over $\mathbb{Z}$ by setting $T_{i_{1}}=T_{i_{2}}$ if $i_{1} \equiv i_{2}(\bmod \ell)$ and $A_{j_{1}}^{\alpha}=A_{j_{2}}^{\alpha}$ if $j_{1} \equiv j_{2}(\bmod h)$.

Define constants $\lambda_{i}^{\alpha}=\alpha\left(T_{i}\right)$. Then $\left[T_{i}, A^{\alpha}\right]=\lambda_{i}^{\alpha} A^{\alpha}$ and $\left[T_{i}, A_{j}^{\alpha}\right]=\lambda_{i}^{\alpha} A_{j+m_{i}}^{\alpha}$.

Consider an automorphism $\sigma$ of $\dot{\mathfrak{g}}$ such that

$$
\sigma=\zeta^{j} \operatorname{Id} \quad \text { on } \dot{\mathfrak{g}}_{j},
$$

where $\zeta \in \mathbb{C}$ is the primitive h-root of 1 . For a root element $A^{\alpha}=\sum_{j=1}^{h} A_{j}^{\alpha}$ we have

$$
\left[T_{i}, \sigma\left(A^{\alpha}\right)\right]=\sigma\left(\left[\sigma^{-1}\left(T_{i}\right), A^{\alpha}\right]\right)=\sigma\left(\left[\zeta^{-m_{i}} T_{i}, A^{\alpha}\right]\right)=\zeta^{-m_{i}} \lambda_{i}^{\alpha} \sigma\left(A^{\alpha}\right)
$$

Thus $\sigma\left(A^{\alpha}\right)=\sum_{j=1}^{h} \zeta^{j} A_{j}^{\alpha}$ is also a root element and $\sigma$ induces an automorphism of $\dot{\Delta}_{s}$. Since all $A_{j}^{\alpha}$ are nonzero then the length of each orbit of $\sigma$ in $\dot{\Delta}_{s}$ is $h$.

We may choose $\ell$ roots $\beta_{1}, \ldots, \beta_{\ell} \in \dot{\Delta}_{s}$ such that $A_{0}^{\beta_{1}}, \ldots, A_{0}^{\beta_{\ell}}$ span $\dot{\mathfrak{g}}_{0}$. Note that if two root elements belong to the same orbit under the action of $\sigma$ then their zero componens are proportional. Therefore every orbit of $\sigma$ contains exactly one of $\beta_{1}, \ldots, \beta_{\ell}$. Consequently, we may choose $\left\{\sigma^{j}\left(A^{\beta_{i}}\right)\right\}, i=1, \ldots, \ell, j=1, \ldots, h$ as our family of the root elements. The set $\left\{A_{j}^{\beta_{i}} \otimes s^{j}, T_{j} \otimes s^{b_{j}}, K_{0}\right\}, i=1, \ldots, \ell, j \in \mathbb{Z}$ forms a basis of $\mathfrak{g}_{s}$.

Remark. The properties of the automorphism $\sigma$ were studied by Kostant in the important paper [Kos]. He proved, in particular, that $\sigma$ acts on the root system $\dot{\Delta}_{s}$ as a Coxeter transformation. Remarkably, the complex coefficients $\lambda_{i}^{\alpha}$ could be interpreted as the orthogonal projections of the roots on a real plane invariant under the Coxeter transformation. These projections were introduced by Coxeter in order to visualize the regular polytopes of higher dimensions. In Chapters 12, 13 of [Co1] and in section 12.5 of [Co2] the case of the root system of type $H_{4}$ is treated, which also gives the answer for $E_{8}$.

Now we shall construct the standard representation $(\varphi, F)$ of $\mathfrak{s}$. The space of this representation (called the Fock space) is the polynomial algebra in the infinitely many variables:

$$
F=\mathbb{C}\left[x_{1}, x_{2}, x_{3}, \ldots\right] .
$$

For $i \geq 1, T_{1-i} \otimes s^{-b_{i}}$ is represented by a multiplication operator:

$$
\varphi\left(T_{1-i} \otimes s^{-b_{i}}\right)=b_{i} x_{i},
$$

$T_{i} \otimes s^{b_{i}}$ is represented by a differentiation operator:

$$
\varphi\left(T_{i} \otimes s^{b_{i}}\right)=\frac{\partial}{\partial x_{i}}
$$


and $\varphi\left(K_{0}\right)=$ Id.

Indeed, the only relation to be checked is

$$
\left[\varphi\left(T_{i} \otimes s^{b_{i}}\right), \varphi\left(T_{1-j} \otimes s^{b_{1-j}}\right)\right]=\frac{1}{h}\left(T_{i} \mid T_{1-j}\right) b_{i} \delta_{b_{i} b_{j}} \varphi\left(K_{0}\right) .
$$

But as it can be easily seen, both expressions are equal to $b_{i} \delta_{i j}$ Id.

One can lift this representation of $\mathfrak{s}$ to the whole $\mathfrak{g}$ using vertex operators. We consider the space $\mathfrak{g}\left[\left[z, z^{-1}\right]\right]$ of formal Laurent series in a variable $z$ with coefficients in $\mathfrak{g}$ and the space $\operatorname{End}(F)\left[\left[z, z^{-1}\right]\right]$ of series with coefficients in $\operatorname{End}(F)$ (see Chapter 2 in [FLM] for details). The adjoint action of $\mathfrak{g}$ on $\mathfrak{g}\left[\left[z, z^{-1}\right]\right]$ is well-defined.

Every representation $\varphi: \mathfrak{g} \rightarrow \operatorname{End}(F)$ defines a homomorphism $\varphi: \mathfrak{g}\left[\left[z, z^{-1}\right]\right] \rightarrow$ $\operatorname{End}(F)\left[\left[z, z^{-1}\right]\right]$.

The following theorem shows how to lift the standard representation of the principal Heisenberg subalgebra to $\mathfrak{g}$ :

Theorem 2 ([Kac] $)$. There exists a representation

$$
\varphi: \mathfrak{g} \rightarrow \operatorname{End}\left(\mathbb{C}\left[x_{1}, x_{2}, \ldots\right]\right)
$$

such that for $i \in \mathbb{N}$

$$
\begin{gathered}
\varphi\left(T_{1-i} \otimes s^{-b_{i}}\right)=b_{i} x_{i}, \\
\varphi\left(T_{i} \otimes s^{b_{i}}\right)=\frac{\partial}{\partial x_{i}}, \\
\varphi\left(K_{0}\right)=\mathrm{Id}, \\
\sum_{j \in \mathbb{Z}} \varphi\left(A_{j}^{\alpha} \otimes s^{j}\right) z^{-j}=A^{\alpha}(z), \text { where } \\
A^{\alpha}(z)=\Lambda_{0}\left(A_{0}^{\alpha} \otimes s^{0}\right) \exp \left(\sum_{i=1}^{\infty} \lambda_{i}^{\alpha} z^{b_{i}} x_{i}\right) \exp \left(-\sum_{i=1}^{\infty} \lambda_{1-i}^{\alpha} \frac{z^{-b_{i}}}{b_{i}} \frac{\partial}{\partial x_{i}}\right) .
\end{gathered}
$$

Since $A_{j}^{\alpha}$ together with $T_{i} \otimes s^{b_{i}}$ and $K_{0}$ span $\mathfrak{g}$ then the above formulas completely determine this representation.

We will use extensively the following Laurent series:

$$
\delta(z)=\sum_{j \in \mathbb{Z}} z^{j} \quad \text { and } \quad D \delta(z)=\sum_{j \in \mathbb{Z}} j z^{j} .
$$

The first of these is the formal analogue of the delta function.

For an element $B=\sum_{j \in \mathbb{Z}_{h}} B_{j} \in \dot{\mathfrak{g}}$ denote by $B(z)$ the formal series $\sum_{j \in \mathbb{Z}} \varphi\left(B_{j} \otimes s^{j}\right) z^{-j} \in$ $\operatorname{End}(F)\left[\left[z, z^{-1}\right]\right]$. 
Corollary 3. Let $A=\sum_{j=1}^{h} A_{j}, B=\sum_{j=1}^{h} B_{j} \in \dot{\mathfrak{g}}$ and let $C^{j}=\left[A_{j}, B\right] \in \dot{\mathfrak{g}}$. Then $\left[A\left(z_{1}\right), B\left(z_{2}\right)\right]=\sum_{j=1}^{h}\left(C^{j}\left(z_{2}\right)+\frac{j}{h}\left(A_{j} \mid B_{-j}\right)\right)\left(\frac{z_{2}}{z_{1}}\right)^{j} \delta\left(\left(\frac{z_{2}}{z_{1}}\right)^{h}\right)+\sum_{j=1}^{h}\left(A_{j} \mid B_{-j}\right)\left(\frac{z_{2}}{z_{1}}\right)^{j} D \delta\left(\left(\frac{z_{2}}{z_{1}}\right)^{h}\right)$.

Proof of the Corollary.

$$
\begin{gathered}
{\left[A\left(z_{1}\right), B\left(z_{2}\right)\right]=\sum_{j \in \mathbb{Z}} \sum_{i \in \mathbb{Z}}\left[\varphi\left(A_{j} \otimes s^{j}\right), \varphi\left(B_{i} \otimes s^{i}\right)\right] z_{1}^{-j} z_{2}^{-i}=} \\
\sum_{j \in \mathbb{Z}} \sum_{i \in \mathbb{Z}}\left\{\varphi\left(\left[A_{j}, B_{i}\right] \otimes s^{j+i}\right)+\frac{1}{h}\left(A_{j} \mid B_{i}\right) j \delta_{j,-i} \varphi\left(K_{0}\right)\right\} z_{1}^{-j} z_{2}^{-i}= \\
\sum_{j \in \mathbb{Z}} \sum_{k=i+j \in \mathbb{Z}} \varphi\left(\left[A_{j}, B_{k-j}\right] \otimes s^{k}\right) z_{1}^{-j} z_{2}^{j-k}+\frac{1}{h} \sum_{j \in \mathbb{Z}}\left(A_{j} \mid B_{-j}\right) j\left(\frac{z_{2}}{z_{1}}\right)^{j}= \\
\sum_{j \in \mathbb{Z}} C^{j}\left(z_{2}\right)\left(\frac{z_{2}}{z_{1}}\right)^{j}+\frac{1}{h} \sum_{j_{1}=1}^{h} \sum_{j=j_{2} \in \mathbb{Z}}\left(A_{j_{1}} \mid B_{-j_{1}}\right)\left(j_{1}+h j_{2}\right)\left(\frac{z_{2}}{z_{1}}\right)^{j_{1}+h j_{2}}= \\
\sum_{j_{1}=1}^{h}\left(C^{j_{1}}\left(z_{2}\right)+\frac{j_{1}}{h}\left(A_{j_{1}} \mid B_{-j_{1}}\right)\right)\left(\frac{z_{2}}{z_{1}}\right)^{j_{1}} \delta\left(\left(\frac{z_{2}}{z_{1}}\right)^{h}\right)+\sum_{j_{1}=1}^{h}\left(A_{j_{1}} \mid B_{-j_{1}}\right)\left(\frac{z_{2}}{z_{1}}\right)^{j_{1}} D \delta\left(\left(\frac{z_{2}}{z_{1}}\right)^{h}\right) .
\end{gathered}
$$

\section{Construction of the vertex operator representation for the toroidal Lie} algebra.

In this section we construct a vertex operator representation for the toroidal Lie algebra that generalizes the principal realization of the basic representation of affine Lie algebra. Again it will be convenient to replace $\mathfrak{g}$ with

$$
\mathfrak{g}_{s}=\sum_{j \in \mathbb{Z}} \dot{\mathfrak{g}}_{j} \otimes s^{j} \mathbb{C}\left[t_{1}^{ \pm}, \ldots, t_{n}^{ \pm}\right] \oplus \mathcal{K} \oplus \mathcal{D}_{+}
$$

with the Lie bracket

$\left[g_{1} \otimes f_{1}(s, \mathbf{t}), g_{2} \otimes f_{2}(s, \mathbf{t})\right]=\left[g_{1}, g_{2}\right] \otimes\left(f_{1} f_{2}\right)+\left(g_{1} \mid g_{2}\right)\left\{\frac{1}{h}\left(s \frac{d}{d s} f_{1}\right) f_{2} K_{0}+\sum_{p=1}^{n} d_{p}\left(f_{1}\right) f_{2} K_{p}\right\}$ 


$$
\left[f_{1}(s, \mathbf{t}) D_{p}, g \otimes f_{2}(s, \mathbf{t})\right]=g \otimes f_{1}\left(d_{p} f_{2}\right)
$$

while the multiplication in $\mathcal{D}_{+}$and its action on $\mathcal{K}$ are the same as in $\mathfrak{g}$. Here and below we make identifications $s^{h r_{0}} \mathbf{t}^{\mathbf{r}} K_{a}=t_{0}^{r_{0}} \mathbf{t}^{\mathbf{r}} K_{a}$ and $s^{h r_{0}} \mathbf{t}^{\mathbf{r}} D_{b}=t_{0}^{r_{0}} \mathbf{t}^{\mathbf{r}} D_{b}, a=0,1, \ldots, n, b=$ $1, \ldots, n$.

The following lemma is an immediate generalization of Lemma 1:

Lemma 4. The Lie algebras $\mathfrak{g}$ and $\mathfrak{g}_{s}$ are isomorphic and the isomorphism is given by

$$
\begin{gathered}
\psi\left(e_{\alpha} \otimes t_{0}^{r_{0}} \mathbf{t}^{\mathbf{r}}\right)=e_{\alpha} \otimes s^{\mathrm{ht}(\alpha)+h r_{0}} \mathbf{t}^{\mathbf{r}} \\
\psi\left(\nu^{-1}(\alpha) \otimes t_{0}^{r_{0}} \mathbf{t}^{\mathbf{r}}\right)=\nu^{-1}(\alpha) \otimes s^{h r_{0}} \mathbf{t}^{\mathbf{r}}+\frac{\mathrm{ht}(\alpha)}{h} s^{h r_{0}} \mathbf{t}^{\mathbf{r}} K_{0}, \\
\psi=\text { Id on } \mathcal{K} \oplus \mathcal{D}_{+} .
\end{gathered}
$$

We shall identify $\mathfrak{g}$ and $\mathfrak{g}_{s}$ using this isomorphism.

The subalgebra $\mathfrak{s}$ with the basis $\left\{T_{i} \otimes s^{b_{i}}, s^{i h} D_{p}, s^{i h} K_{p}, K_{0}\right\}, i \in \mathbb{Z}, \quad p=1, \ldots, n$ is the principal (degenerate) Heisenberg subalgebra of $\mathfrak{g}$.

Indeed, $K_{0}$ is its central element and the multiplication in $\mathfrak{s}$ is given by

$$
\begin{gathered}
{\left[T_{i} \otimes s^{b_{i}}, T_{1-j} \otimes s^{-b_{j}}\right]=b_{i} \delta_{i j} K_{0},} \\
{\left[s^{i h} D_{a}, s^{j h} K_{b}\right]=\delta_{a b} i s^{(i+j) h} K_{0}=i \delta_{a b} \delta_{i,-j} K_{0},} \\
{\left[s^{j h} D_{p}, T_{i} \otimes s^{b_{i}}\right]=0,\left[s^{i h} D_{a}, s^{j h} D_{b}\right]=0,} \\
{\left[T_{i} \otimes s^{b_{i}}, s^{j h} K_{p}\right]=0,\left[s^{i h} K_{a}, s^{j h} K_{b}\right]=0,}
\end{gathered}
$$

where $i, j \in \mathbb{Z}, a, b, p=1, \ldots, n$. This subalgebra is degenerate as a Heisenbeg algebra since $\left[D_{p}, K_{p}\right]=0$.

The Heisenberg algebra $\mathfrak{s}$ can be represented on the Fock space

$$
F=\mathbb{C}\left[q_{p}^{ \pm}, x_{i}, u_{p i}, v_{p i}\right]_{i \in \mathbb{N}}^{p=1, \ldots, n}
$$

by differentiation and multiplication operators:

$$
\begin{gathered}
\varphi\left(T_{i} \otimes s^{b_{i}}\right)=\frac{\partial}{\partial x_{i}}, \varphi\left(T_{1-i} \otimes s^{-b_{i}}\right)=b_{i} x_{i}, \\
\varphi\left(s^{i h} D_{p}\right)=\frac{\partial}{\partial u_{p i}}, \varphi\left(s^{-i h} D_{p}\right)=i v_{p i}, \\
\varphi\left(s^{i h} K_{p}\right)=\frac{\partial}{\partial v_{p i}}, \varphi\left(s^{-i h} K_{p}\right)=i u_{p i},
\end{gathered}
$$




$$
\begin{gathered}
\varphi\left(D_{p}\right)=q_{p} \frac{\partial}{\partial q_{p}}, \varphi\left(K_{p}\right)=0, \\
\varphi\left(K_{0}\right)=\mathrm{Id},
\end{gathered}
$$

where $i \geq 1$ and $p=1, \ldots, n$. Our goal is to extend this representation of $\mathfrak{s}$ to $\mathfrak{g}$. Consider the following elements of $\mathfrak{g}\left[\left[z, z^{-1}\right]\right]$ :

$$
\sum_{j \in \mathbb{Z}} s^{j h} \mathbf{t}^{\mathbf{r}} K_{0} z^{-j h} \text { and } \sum_{j \in \mathbb{Z}} A_{j}^{\alpha} \otimes s^{j} \mathbf{t}^{\mathbf{r}} z^{-j} .
$$

Note that

$$
\begin{gathered}
{\left[T_{i} \otimes s^{b_{i}}, \sum_{j \in \mathbb{Z}} s^{j h} \mathbf{t}^{\mathbf{r}} K_{0} z^{-j h}\right]=0,} \\
{\left[s^{i h} K_{p}, \sum_{j \in \mathbb{Z}} s^{j h} \mathbf{t}^{\mathbf{r}} K_{0} z^{-j h}\right]=0,} \\
{\left[s^{i h} D_{p}, \sum_{j \in \mathbb{Z}} s^{j h} \mathbf{t}^{\mathbf{r}} K_{0} z^{-j h}\right]=\sum_{j \in \mathbb{Z}} r_{p} s^{(i+j) h} \mathbf{t}^{\mathbf{r}} K_{0} z^{-j h}=} \\
\sum_{k=i+j \in \mathbb{Z}} r_{p} s^{k h} \mathbf{t}^{\mathbf{r}} K_{0} z^{-k h+i h}=r_{p} z^{i h} \sum_{j \in \mathbb{Z}} s^{j h} \mathbf{t}^{\mathbf{r}} K_{0} z^{-j h} .
\end{gathered}
$$

The theory of vertex operators suggests (see Lemma 14.5 in [Kac]) that $\sum_{j \in \mathbb{Z}} s^{j h} \mathbf{t}^{\mathbf{r}} K_{0} z^{-j h}$ should be represented by

$$
K_{0}(z, \mathbf{r})=\mathbf{q}^{\mathbf{r}} \exp \left(\sum_{p=1}^{n} r_{p} \sum_{j \geq 1} z^{j h} u_{p j}\right) \exp \left(-\sum_{p=1}^{n} r_{p} \sum_{j \geq 1} \frac{z^{-j h}}{j} \frac{\partial}{\partial v_{p j}}\right) .
$$

Here $\mathbf{q}^{\mathbf{r}}=q_{1}^{r_{1}} \ldots q_{n}^{r_{n}}$.

In a similar way the commutator relations

$$
\begin{gathered}
{\left[T_{i} \otimes s^{b_{i}}, \sum_{j \in \mathbb{Z}} A_{j}^{\alpha} \otimes s^{j} \mathbf{t}^{\mathbf{r}} z^{-j}\right]=\sum_{j \in \mathbb{Z}} \lambda_{i}^{\alpha} A_{j+b_{i}}^{\alpha} \otimes s^{j+b_{i}} \mathbf{t}^{\mathbf{r}} z^{-j}=} \\
\lambda_{i}^{\alpha} \sum_{k=j+b_{i} \in \mathbb{Z}} A_{k}^{\alpha} \otimes s^{k} \mathbf{t}^{\mathbf{r}} z^{-k+b_{i}}=\lambda_{i}^{\alpha} z^{b_{i}} \sum_{j \in \mathbb{Z}} A_{j}^{\alpha} \otimes s^{j} \mathbf{t}^{\mathbf{r}} z^{-j}, \\
{\left[s^{i h} K_{p}, \sum_{j \in \mathbb{Z}} A_{j}^{\alpha} \otimes s^{j} \mathbf{t}^{\mathbf{r}} z^{-j}\right]=0,} \\
{\left[s^{i h} D_{p}, \sum_{j \in \mathbb{Z}} A_{j}^{\alpha} \otimes s^{j} \mathbf{t}^{\mathbf{r}} z^{-j}\right]=\sum_{j \in \mathbb{Z}} r_{p} A_{j}^{\alpha} \otimes s^{j+i h} \mathbf{t}^{\mathbf{r}} z^{-j}=}
\end{gathered}
$$




$$
r_{p} \sum_{k=j+i h \in \mathbb{Z}} A_{j}^{\alpha} \otimes s^{k} \mathbf{t}^{\mathbf{r}} z^{-k+i h}=r_{p} z^{i h} \sum_{j \in \mathbb{Z}} A_{j}^{\alpha} \otimes s^{j} \mathbf{t}^{\mathbf{r}} z^{-j}
$$

suggest that $\sum_{j \in \mathbb{Z}} A_{j}^{\alpha} \otimes s^{j} \mathbf{t}^{\mathbf{r}} z^{-j}$ should be represented by

$$
\begin{gathered}
A^{\alpha}(z, \mathbf{r})= \\
\mathbf{q}^{\mathbf{r}} \Lambda_{0}\left(A_{0}^{\alpha} \otimes s^{0}\right) \exp \left(\sum_{i=1}^{\infty} \lambda_{i}^{\alpha} z^{b_{i}} x_{i}\right) \exp \left(-\sum_{i=1}^{\infty} \lambda_{1-i}^{\alpha} \frac{z^{-b_{i}}}{b_{i}} \frac{\partial}{\partial x_{i}}\right) \times \\
\times \exp \left(\sum_{p=1}^{n} r_{p} \sum_{j \in \mathbb{Z}} z^{j h} u_{p j}\right) \exp \left(-\sum_{p=1}^{n} r_{p} \sum_{j \in \mathbb{Z}} \frac{z^{-j h}}{j} \frac{\partial}{\partial v_{p j}}\right)= \\
A^{\alpha}(z) K_{0}(z, \mathbf{r}) .
\end{gathered}
$$

The last formula hints that for $B \in \dot{\mathfrak{g}}$ we may try to represent $\sum_{j \in \mathbb{Z}} B_{j} \otimes s^{j} \mathbf{t}^{\mathbf{r}} z^{-j}$ by

$$
B(z, \mathbf{r})=B(z) K_{0}(z, \mathbf{r})
$$

Note that $B(z)$ is known from the affine case (Theorem 2).

In fact, we shall see that the same approach is valid even for $\mathcal{K}$ and $\mathcal{D}_{+}$, but for these we should first discuss the concept of the normal ordering.

Let $X(z)=\sum_{i \in \mathbb{Z}} X_{i} z^{i}, Y(z)=\sum_{j \in \mathbb{Z}} Y_{j} z^{j} \in \operatorname{End}(F)\left[\left[z, z^{-1}\right]\right]$. We say that the product $X(z) Y(z)$ exists if for every $k \in \mathbb{Z}$ and for every $v \in F$ the sum

$$
\sum_{i \in \mathbb{Z}} X_{i} Y_{k-i} v
$$

has finitely many nonzero terms. If this is the case then

$$
X(z) Y(z)=\sum_{k \in \mathbb{Z}}\left(\sum_{i \in \mathbb{Z}} X_{i} Y_{k-i}\right) z^{k}
$$

It is possible that $X(z) Y(z)$ exists while $Y(z) X(z)$ does not. For example, this happens for

$$
X(z)=\sum_{i \geq 1} x_{i} z^{i} \text { and } Y(z)=\sum_{i \geq 1} \frac{\partial}{\partial x_{i}} z^{-i}
$$

To improve the situation we define the normal ordering for the product of operators on $F$. For a pseudodifferential operator $P \in \operatorname{End}(F)$ the normal ordering $: x_{i} P$ : is defined as $x_{i} P$, while the normal ordering of the product $: \frac{\partial}{\partial x_{i}} P$ : is defined as $P \frac{\partial}{\partial x_{i}}$. Note that for $X(z)$ and $Y(z)$ from the above example $: X(z) Y(z):=: Y(z) X(z):=X(z) Y(z)$. 
From the action of the principle Heisenberg subalgebra on the Fock space we see that the series $\sum_{j \in \mathbb{Z}} s^{j h} K_{p} z^{-j h}$ and $\sum_{j \in \mathbb{Z}} s^{j h} D_{p} z^{-j h}$ are represented by

$$
K_{p}(z)=\sum_{i \geq 1} i u_{p i} z^{i h}+\sum_{i \geq 1} \frac{\partial}{\partial v_{p i}} z^{-i h}
$$

and

$$
D_{p}(z)=\sum_{i \geq 1} i v_{p i} z^{i h}+q_{p} \frac{\partial}{\partial q_{p}}+\sum_{i \geq 1} \frac{\partial}{\partial u_{p i}} z^{-i h} .
$$

Using the analogy with $A^{\alpha}(z, \mathbf{r})$ we shall represent $\sum_{j \in \mathbb{Z}} s^{j h} \mathbf{t}^{\mathbf{r}} K_{p} z^{-j h}$ by

$$
K_{p}(z, \mathbf{r})=K_{p}(z) K_{0}(z, \mathbf{r})
$$

and $\sum_{j \in \mathbb{Z}} s^{j h} \mathbf{t}^{\mathbf{r}} D_{p} z^{-j h}$ by

$$
D_{p}(z, \mathbf{r})=: D_{p}(z) K_{0}(z, \mathbf{r}): .
$$

Note that we use the normal ordering in the case of $D_{p}(z, \mathbf{r})$ in order for the product to exist.

We summarize this discussion in our main theorem:

Theorem 5. There exists a representation $\varphi$ of the toroidal Lie algebra

$$
\dot{\mathfrak{g}} \otimes \mathbb{C}\left[s^{ \pm}, t_{1}^{ \pm}, \ldots, t_{n}^{ \pm}\right] \oplus \mathcal{K} \oplus \mathcal{D}_{+}
$$

on the Fock space

$$
F=\mathbb{C}\left[q_{p}^{ \pm}, x_{i}, u_{p i}, v_{p i}\right]_{i \in \mathbb{N}}^{p=1, \ldots, n}
$$

such that for $i=1, \ldots, \ell, p=1, \ldots, n, \alpha \in \dot{\Delta}_{s}, \mathbf{r} \in \mathbb{Z}^{n}$

$$
\begin{gathered}
\sum_{j \in \mathbb{Z}} \varphi\left(s^{j h} \mathbf{t}^{\mathbf{r}} K_{0}\right) z^{-j h}=K_{0}(z, \mathbf{r})=\mathbf{q}^{\mathbf{r}} \exp \left(\sum_{p=1}^{n} r_{p} \sum_{j \geq 1} z^{j h} u_{p j}\right) \exp \left(-\sum_{p=1}^{n} r_{p} \sum_{j \geq 1} \frac{z^{-j h}}{j} \frac{\partial}{\partial v_{p j}}\right), \\
\sum_{j \in \mathbb{Z}} \varphi\left(T_{i} \otimes s^{m_{i}+j h} \mathbf{t}^{\mathbf{r}}\right) z^{-m_{i}-j h}=T_{i}(z, \mathbf{r})= \\
\left\{\sum_{j \geq 1}\left(j h-m_{i}\right) z^{j h-m_{i}} x_{j \ell+1-i}+\sum_{j \geq 0} z^{-j h-m_{i}} \frac{\partial}{\partial x_{j \ell+i}}\right\} K_{0}(z, \mathbf{r}) \\
\sum_{j \in \mathbb{Z}} \varphi\left(A_{j}^{\alpha} \otimes s^{j} \mathbf{t}^{\mathbf{r}}\right) z^{-j}=A^{\alpha}(z, \mathbf{r})=
\end{gathered}
$$




$$
\begin{gathered}
\Lambda_{0}\left(A_{0}^{\alpha} \otimes s^{0}\right) \exp \left(\sum_{i=1}^{\infty} \lambda_{i}^{\alpha} z^{b_{i}} x_{i}\right) \exp \left(-\sum_{i=1}^{\infty} \lambda_{1-i}^{\alpha} \frac{z^{-b_{i}}}{b_{i}} \frac{\partial}{\partial x_{i}}\right) K_{0}(z, \mathbf{r}), \\
\sum_{j \in \mathbb{Z}} \varphi\left(s^{j h} \mathbf{t}^{\mathbf{r}} K_{p}\right) z^{-j h}=K_{p}(z, \mathbf{r})=\left\{\sum_{i \geq 1} i u_{p i} z^{i h}+\sum_{i \geq 1} \frac{\partial}{\partial v_{p i}} z^{-i h}\right\} K_{0}(z, \mathbf{r}), \\
\sum_{j \in \mathbb{Z}} \varphi\left(s^{j h} \mathbf{t}^{\mathbf{r}} D_{p}\right) z^{-j h}=D_{p}(z, \mathbf{r})=:\left\{\sum_{i \geq 1} i v_{p i} z^{i h}+q_{p} \frac{\partial}{\partial q_{p}}+\sum_{i \geq 1} \frac{\partial}{\partial u_{p i}} z^{-i h}\right\} K_{0}(z, \mathbf{r}): .
\end{gathered}
$$

\section{Proof of the main theorem.}

First of all, let us check that formulas (3.1) - (3.5) define a linear map $\varphi: \mathfrak{g} \rightarrow \operatorname{End}(F)$. The linear dependencies between the momenta at the left hand side are of the form

$$
\varphi\left(A_{j}^{\sigma^{k}(\alpha)} \otimes s^{j} \mathbf{t}^{\mathbf{r}}\right)=\zeta^{k j} \varphi\left(A_{j}^{\alpha} \otimes s^{j} \mathbf{t}^{\mathbf{r}}\right)
$$

and

$$
j \varphi\left(s^{j h} \mathbf{t}^{\mathbf{r}} K_{0}\right)+\sum_{p=1}^{n} r_{p} \varphi\left(s^{j h} \mathbf{t}^{\mathbf{r}} K_{p}\right)=0 .
$$

These dependencies extend to the following relations for the corresponding series:

$$
\sum_{j \in \mathbb{Z}} \varphi\left(A_{j}^{\sigma^{k}(\alpha)} \otimes s^{j} \mathbf{t}^{\mathbf{r}}\right) z^{-j}=\sum_{j \in \mathbb{Z}} \varphi\left(A_{j}^{\alpha} \otimes s^{j} \mathbf{t}^{\mathbf{r}}\right)\left(\zeta^{-k} z\right)^{-j}
$$

and

$$
-\frac{1}{h} D_{z} \sum_{j \in \mathbb{Z}} \varphi\left(s^{j h} \mathbf{t}^{\mathbf{r}} K_{0}\right) z^{-j h}+\sum_{p=1}^{n} r_{p} \sum_{j \in \mathbb{Z}} \varphi\left(s^{j h} \mathbf{t}^{\mathbf{r}} K_{p}\right) z^{-j h}=0,
$$

where $D_{z}=z \frac{\partial}{\partial z}$. We have to show that the same relations hold for the right hand sides of (3.1) - (3.5). Indeed, noting that $\lambda_{i}^{\sigma^{k}(\alpha)}=\zeta^{-k m_{i}} \lambda_{i}^{\alpha}$ we obtain

$$
\begin{aligned}
& A^{\sigma^{k}(\alpha)}(z, \mathbf{r})=\Lambda_{0}\left(A_{0}^{\sigma^{k}(\alpha)}\right) \exp \left(\sum_{i=1}^{\infty} \lambda_{i}^{\alpha} \zeta^{-k m_{i}} z^{b_{i}} x_{i}\right) \exp \left(-\sum_{i=1}^{\infty} \lambda_{1-i}^{\alpha} \zeta^{k m_{i}} \frac{z^{-b_{i}}}{b_{i}} \frac{\partial}{\partial x_{i}}\right) K_{0}(z, \mathbf{r})= \\
& \Lambda_{0}\left(A_{0}^{\alpha}\right) \exp \left(\sum_{i=1}^{\infty} \lambda_{i}^{\alpha}\left(\zeta^{-k} z\right)^{b_{i}} x_{i}\right) \exp \left(-\sum_{i=1}^{\infty} \lambda_{1-i}^{\alpha} \frac{\left(\zeta^{-k} z\right)^{-b_{i}}}{b_{i}} \frac{\partial}{\partial x_{i}}\right) K_{0}\left(\zeta^{-k} z, \mathbf{r}\right)=A^{\alpha}\left(\zeta^{-k} z, \mathbf{r}\right) .
\end{aligned}
$$

The verification of the second relation is also straightforward:

$$
D_{z} K_{0}(z, \mathbf{r})=D_{z} \mathbf{q}^{\mathbf{r}} \exp \left(\sum_{p=1}^{n} r_{p} \sum_{j \geq 1} z^{j h} u_{p j}\right) \exp \left(-\sum_{p=1}^{n} r_{p} \sum_{j \geq 1} \frac{z^{-j h}}{j} \frac{\partial}{\partial v_{p j}}\right)=
$$




$$
\begin{gathered}
\sum_{p=1}^{n} r_{p}\left\{\sum_{j \geq 1} z^{j h} j h u_{p j}+\sum_{j \geq 1} z^{-j h} h \frac{\partial}{\partial v_{p j}}\right\} K_{0}(z, \mathbf{r})= \\
h \sum_{p=1}^{n} r_{p} K_{p}(z, \mathbf{r}) .
\end{gathered}
$$

Observing that the momenta of the series in the left hand sides of (3.1) - (3.5) span $\mathfrak{g}$ we conclude that the linear map $\varphi: \mathfrak{g} \rightarrow \operatorname{End}(F)$ is well-defined. We need to show now that $\varphi$ is a homomorphism of Lie algebras.

The following Lemma and its Corollary which we state without proof are very useful for the computations with formal series.

Lemma 6 (cf. Proposition 2.2.2 in $[\mathrm{FLM}]$ ). Let

$$
\delta(z)=\sum_{k \in \mathbb{Z}} z^{k} \quad \text { and } \quad D \delta(z)=D_{z} \delta(z)=\sum_{k \in \mathbb{Z}} k z^{k}
$$

If the products in the left hand sides exist then the following equalities hold:

$$
\begin{gathered}
X\left(z_{1}\right) \delta\left(\left(\frac{z_{2}}{z_{1}}\right)\right)=X\left(z_{2}\right) \delta\left(\left(\frac{z_{2}}{z_{1}}\right)\right), \\
X\left(z_{1}\right) D \delta\left(\left(\frac{z_{2}}{z_{1}}\right)\right)=X\left(z_{2}\right) D \delta\left(\left(\frac{z_{2}}{z_{1}}\right)\right)+D_{z_{2}}\left(X\left(z_{2}\right)\right) \delta\left(\left(\frac{z_{2}}{z_{1}}\right)\right),
\end{gathered}
$$

Corollary 7. Let $X(z)=Y\left(z^{h}\right)$. The following equalities hold provided the products in the left hand sides exist:

$$
\begin{gathered}
X\left(z_{1}\right) \delta\left(\left(\frac{z_{2}}{z_{1}}\right)^{h}\right)=X\left(z_{2}\right) \delta\left(\left(\frac{z_{2}}{z_{1}}\right)^{h}\right), \\
X\left(z_{1}\right) D \delta\left(\left(\frac{z_{2}}{z_{1}}\right)^{h}\right)=X\left(z_{2}\right) D \delta\left(\left(\frac{z_{2}}{z_{1}}\right)^{h}\right)+\frac{1}{h} D_{z_{2}}\left(X\left(z_{2}\right)\right) \delta\left(\left(\frac{z_{2}}{z_{1}}\right)^{h}\right),
\end{gathered}
$$

Note that $A^{\alpha}(z, \mathbf{r})=A^{\alpha}(z) K_{0}(z, \mathbf{r})$ and $T_{i}(z, \mathbf{r})=T_{i}(z) K_{0}(z, \mathbf{r})$, hence for every $B \in \dot{\mathfrak{g}}$ we have $B(z, \mathbf{r})=B(z) K_{0}(z, \mathbf{r})$.

In order to verify the commutator relations between the elements of $\sum_{j \in \mathbb{Z}} \dot{\mathfrak{g}}_{j} \otimes s^{j} \mathbb{C}\left[t_{1}^{ \pm}, \ldots, t_{n}^{ \pm}\right]$we have to show that for every $A, B \in \dot{\mathfrak{g}}$

$$
\sum_{j, i \in \mathbb{Z}} \varphi\left(\left[A_{j} \otimes s^{j} \mathbf{t}^{\mathbf{r}}, B_{i} \otimes s^{i} \mathbf{t}^{\mathbf{m}}\right]\right) z_{1}^{-j} z_{2}^{-i}=\sum_{j, i \in \mathbb{Z}}\left[\varphi\left(A_{j} \otimes s^{j} \mathbf{t}^{\mathbf{r}}\right), \varphi\left(B_{i} \otimes s^{i} \mathbf{t}^{\mathbf{m}}\right)\right] z_{1}^{-j} z_{2}^{-i}
$$


Indeed, let $C^{j}=\left[A_{j}, B\right]$. Then

$$
\begin{aligned}
& \sum_{j, i \in \mathbb{Z}} \varphi\left(\left[A_{j} \otimes s^{j} \mathbf{t}^{\mathbf{r}}, B_{i} \otimes s^{i} \mathbf{t}^{\mathbf{m}}\right]\right) z_{1}^{-j} z_{2}^{-i}= \\
& =\sum_{j, i \in \mathbb{Z}} \varphi\left(\left[A_{j}, B_{i}\right] \otimes s^{j+i} \mathbf{t}^{\mathbf{r}+\mathbf{m}}\right) z_{1}^{-j} z_{2}^{-i}+ \\
& \sum_{j, i \in \mathbb{Z}}\left(A_{j} \mid B_{i}\right) \varphi\left(s^{j+i} \mathbf{t}^{\mathbf{r}+\mathbf{m}}\left(\frac{j}{h} K_{0}+\sum_{p=1}^{n} r_{p} K_{p}\right)\right)= \\
& =\sum_{j \in \mathbb{Z}} \sum_{k=j+i \in \mathbb{Z}} \varphi\left(\left[A_{j}, B_{k-j}\right] \otimes s^{k} \mathbf{t}^{\mathbf{r}+\mathbf{m}}\right) z_{1}^{-j} z_{2}^{j-k}+ \\
& \sum_{j_{1}=1}^{h} \sum_{\substack{j_{2} \in \mathbb{Z} \\
j=j_{1}+h j_{2}}} \sum_{\substack{i_{2} \in \mathbb{Z} \\
i=-j_{1}+h i_{2}}}\left(A_{j_{1}} \mid B_{-j_{1}}\right) \frac{j_{1}+h j_{2}}{h} \varphi\left(s^{h\left(j_{2}+i_{2}\right)} \mathbf{t}^{\mathbf{r}+\mathbf{m}} K_{0}\right) z_{1}^{-j_{1}-h j_{2}} z_{2}^{j_{1}-h i_{2}}+ \\
& \sum_{j_{1}=1}^{h} \sum_{\substack{j_{2} \in \mathbb{Z} \\
j=j_{1}+h j_{2}}} \sum_{\substack{i=-j_{1} \in \mathbb{Z}_{1} \\
i i_{2}}}\left(A_{j_{1}} \mid B_{-j_{1}}\right) \sum_{p=1}^{n} r_{p} \varphi\left(s^{h\left(j_{2}+i_{2}\right)} \mathbf{t}^{\mathbf{r}+\mathbf{m}} K_{p}\right) z_{1}^{-j_{1}-h j_{2}} z_{2}^{j_{1}-h i_{2}}= \\
& =\sum_{j_{1}=1}^{h} \sum_{j_{2} \in \mathbb{Z}} \sum_{k \in \mathbb{Z}} \varphi\left(C_{k}^{j_{1}} \otimes s^{k} \mathbf{t}^{\mathbf{r}+\mathbf{m}}\right) z_{2}^{-k}\left(\frac{z_{2}}{z_{1}}\right)^{j_{1}}\left(\frac{z_{2}}{z_{1}}\right)^{h j_{2}}+ \\
& \sum_{j_{1}=1}^{h} \sum_{j_{2} \in \mathbb{Z}} \sum_{k=j_{2}+i_{2} \in \mathbb{Z}}\left(A_{j_{1}} \mid B_{-j_{1}}\right) \frac{j_{1}+h j_{2}}{h} \varphi\left(s^{h k} \mathbf{t}^{\mathbf{r}+\mathbf{m}} K_{0}\right) z_{1}^{-j_{1}-h j_{2}} z_{2}^{j_{1}+h j_{2}-h k}+ \\
& \sum_{j_{1}=1}^{h} \sum_{j_{2} \in \mathbb{Z}} \sum_{k=j_{2}+i_{2} \in \mathbb{Z}}\left(A_{j_{1}} \mid B_{-j_{1}}\right) \sum_{p=1}^{n} r_{p} \varphi\left(s^{h k} \mathbf{t}^{\mathbf{r}+\mathbf{m}} K_{p}\right) z_{1}^{-j_{1}-h j_{2}} z_{2}^{j_{1}+h j_{2}-h k}= \\
& =\sum_{j_{1}=1}^{h} C^{j_{1}}\left(z_{2}, \mathbf{r}+\mathbf{m}\right)\left(\frac{z_{2}}{z_{1}}\right)^{j_{1}} \delta\left(\left(\frac{z_{2}}{z_{1}}\right)^{h}\right)+ \\
& \frac{1}{h} \sum_{j_{1}=1}^{h} j_{1}\left(A_{j_{1}} \mid B_{-j_{1}}\right) K_{0}\left(z_{2}, \mathbf{r}+\mathbf{m}\right)\left(\frac{z_{2}}{z_{1}}\right)^{j_{1}} \delta\left(\left(\frac{z_{2}}{z_{1}}\right)^{h}\right)+ \\
& \sum_{p=1}^{n} r_{p} \sum_{j_{1}=1}^{h}\left(A_{j_{1}} \mid B_{-j_{1}}\right) K_{p}\left(z_{2}, \mathbf{r}+\mathbf{m}\right)\left(\frac{z_{2}}{z_{1}}\right)^{j_{1}} \delta\left(\left(\frac{z_{2}}{z_{1}}\right)^{h}\right)+ \\
& \sum_{j_{1}=1}^{h}\left(A_{j_{1}} \mid B_{-j_{1}}\right) K_{0}\left(z_{2}, \mathbf{r}+\mathbf{m}\right)\left(\frac{z_{2}}{z_{1}}\right)^{j_{1}} D \delta\left(\left(\frac{z_{2}}{z_{1}}\right)^{h}\right) \text {. }
\end{aligned}
$$

For the right hand side of (4.1) we use Corollary 3, Corollary 7 and the fact that 


$$
\begin{aligned}
& K_{0}(z, \mathbf{r}) K_{0}(z, \mathbf{m})=K_{0}(z, \mathbf{r}+\mathbf{m}): \\
& \sum_{j, i \in \mathbb{Z}}\left[\varphi\left(A_{j} \otimes s^{j} \mathbf{t}^{\mathbf{r}}\right), \varphi\left(B_{i} \otimes s^{i} \mathbf{t}^{\mathbf{m}}\right)\right] z_{1}^{-j} z_{2}^{-i}=\left[A\left(z_{1}, \mathbf{r}\right), B\left(z_{2}, \mathbf{m}\right)\right]= \\
& =\left[A\left(z_{1}\right) K_{0}\left(z_{1}, \mathbf{r}\right), B\left(z_{2}\right) K_{0}\left(z_{2}, \mathbf{m}\right)\right]=\left[A\left(z_{1}\right), B\left(z_{2}\right)\right] K_{0}\left(z_{1}, \mathbf{r}\right) K_{0}\left(z_{2}, \mathbf{m}\right)= \\
& =\sum_{j=1}^{h} C^{j}\left(z_{2}\right)\left(\frac{z_{2}}{z_{1}}\right)^{j} \delta\left(\left(\frac{z_{2}}{z_{1}}\right)^{h}\right) K_{0}\left(z_{1}, \mathbf{r}\right) K_{0}\left(z_{2}, \mathbf{m}\right)+ \\
& \frac{1}{h} \sum_{j=1}^{h} j\left(A_{j} \mid B_{-j}\right)\left(\frac{z_{2}}{z_{1}}\right)^{j} \delta\left(\left(\frac{z_{2}}{z_{1}}\right)^{h}\right) K_{0}\left(z_{1}, \mathbf{r}\right) K_{0}\left(z_{2}, \mathbf{m}\right)+ \\
& \sum_{j=1}^{h}\left(A_{j} \mid B_{-j}\right)\left(\frac{z_{2}}{z_{1}}\right)^{j} D \delta\left(\left(\frac{z_{2}}{z_{1}}\right)^{h}\right) K_{0}\left(z_{1}, \mathbf{r}\right) K_{0}\left(z_{2}, \mathbf{m}\right)= \\
& =\sum_{j=1}^{h} C^{j}\left(z_{2}\right)\left(\frac{z_{2}}{z_{1}}\right)^{j} \delta\left(\left(\frac{z_{2}}{z_{1}}\right)^{h}\right) K_{0}\left(z_{2}, \mathbf{r}\right) K_{0}\left(z_{2}, \mathbf{m}\right)+ \\
& \frac{1}{h} \sum_{j=1}^{h} j\left(A_{j} \mid B_{-j}\right)\left(\frac{z_{2}}{z_{1}}\right)^{j} \delta\left(\left(\frac{z_{2}}{z_{1}}\right)^{h}\right) K_{0}\left(z_{2}, \mathbf{r}\right) K_{0}\left(z_{2}, \mathbf{m}\right)+ \\
& \sum_{j=1}^{h}\left(A_{j} \mid B_{-j}\right)\left(\frac{z_{2}}{z_{1}}\right)^{j} D \delta\left(\left(\frac{z_{2}}{z_{1}}\right)^{h}\right) K_{0}\left(z_{2}, \mathbf{r}\right) K_{0}\left(z_{2}, \mathbf{m}\right)+ \\
& \sum_{j=1}^{h}\left(A_{j} \mid B_{-j}\right)\left(\frac{z_{2}}{z_{1}}\right)^{j} \delta\left(\left(\frac{z_{2}}{z_{1}}\right)^{h}\right) \frac{1}{h} D_{z_{2}}\left(K_{0}\left(z_{2}, \mathbf{r}\right)\right) K_{0}\left(z_{2}, \mathbf{m}\right)= \\
& =\sum_{j=1}^{h} C^{j}\left(z_{2}\right)\left(\frac{z_{2}}{z_{1}}\right)^{j} \delta\left(\left(\frac{z_{2}}{z_{1}}\right)^{h}\right) K_{0}\left(z_{2}, \mathbf{r}+\mathbf{m}\right)+ \\
& \frac{1}{h} \sum_{j=1}^{h} j\left(A_{j} \mid B_{-j}\right)\left(\frac{z_{2}}{z_{1}}\right)^{j} \delta\left(\left(\frac{z_{2}}{z_{1}}\right)^{h}\right) K_{0}\left(z_{2}, \mathbf{r}+\mathbf{m}\right)+ \\
& \sum_{j=1}^{h}\left(A_{j} \mid B_{-j}\right)\left(\frac{z_{2}}{z_{1}}\right)^{j} D \delta\left(\left(\frac{z_{2}}{z_{1}}\right)^{h}\right) K_{0}\left(z_{2}, \mathbf{r}+\mathbf{m}\right)+ \\
& \sum_{j=1}^{h}\left(A_{j} \mid B_{-j}\right)\left(\frac{z_{2}}{z_{1}}\right)^{j} \delta\left(\left(\frac{z_{2}}{z_{1}}\right)^{h}\right) \sum_{p=1}^{n} r_{p} K_{p}\left(z_{2}\right) K_{0}\left(z_{2}, \mathbf{r}\right) K_{0}\left(z_{2}, \mathbf{m}\right)=
\end{aligned}
$$




$$
\begin{aligned}
= & \sum_{j=1}^{h} C^{j}\left(z_{2}\right)\left(\frac{z_{2}}{z_{1}}\right)^{j} \delta\left(\left(\frac{z_{2}}{z_{1}}\right)^{h}\right) K_{0}\left(z_{2}, \mathbf{r}+\mathbf{m}\right)+ \\
& \frac{1}{h} \sum_{j=1}^{h} j\left(A_{j} \mid B_{-j}\right)\left(\frac{z_{2}}{z_{1}}\right)^{j} \delta\left(\left(\frac{z_{2}}{z_{1}}\right)^{h}\right) K_{0}\left(z_{2}, \mathbf{r}+\mathbf{m}\right)+ \\
& \sum_{p=1}^{n} r_{p} \sum_{j=1}^{h}\left(A_{j} \mid B_{-j}\right)\left(\frac{z_{2}}{z_{1}}\right)^{j} \delta\left(\left(\frac{z_{2}}{z_{1}}\right)^{h}\right) K_{p}\left(z_{2}, \mathbf{r}+\mathbf{m}\right)+ \\
& \sum_{j=1}^{h}\left(A_{j} \mid B_{-j}\right)\left(\frac{z_{2}}{z_{1}}\right)^{j} D \delta\left(\left(\frac{z_{2}}{z_{1}}\right)^{h}\right) K_{0}\left(z_{2}, \mathbf{r}+\mathbf{m}\right) .
\end{aligned}
$$

Thus (4.1) holds.

It is easy to see that operators $\varphi\left(s^{j h} t^{\mathbf{r}} K_{p}\right), p=0,1, \ldots, n$ commute with $\varphi\left(\sum_{j \in \mathbb{Z}} \dot{\mathfrak{g}}_{j} \otimes s^{j} \mathbb{C}\left[t_{1}^{ \pm}, \ldots, t_{n}^{ \pm}\right] \oplus \mathcal{K}\right)$. This follows from the obvious equalities:

$$
\begin{gathered}
{\left[K_{p}\left(z_{1}, \mathbf{r}\right), A^{\alpha}\left(z_{2}, \mathbf{m}\right)\right]=\left[K_{p}\left(z_{1}, \mathbf{r}\right), T_{i}\left(z_{2}, \mathbf{m}\right)\right]=0} \\
{\left[K_{a}\left(z_{1}, \mathbf{r}\right), K_{b}\left(z_{2}, \mathbf{m}\right)\right]=0, \quad p, a, b=0,1, \ldots, n .}
\end{gathered}
$$

In order to check the action of $\mathcal{D}_{+}$on $\sum_{j \in \mathbb{Z}} \dot{\mathfrak{g}}_{j} \otimes s^{j} \mathbb{C}\left[t_{1}^{ \pm}, \ldots, t_{n}^{ \pm}\right]$it is sufficient to verify the following equality for the generating series:

$$
\sum_{j, i \in \mathbb{Z}} \varphi\left(\left[s^{j h} \mathbf{t}^{\mathbf{r}} D_{p}, s^{i} \mathbf{t}^{\mathbf{m}} A_{i}^{\alpha}\right]\right) z_{1}^{-j h} z_{2}^{-i}=\sum_{j, i \in \mathbb{Z}}\left[\varphi\left(s^{j h} \mathbf{t}^{\mathbf{r}} D_{p}\right), \varphi\left(A_{i}^{\alpha} \otimes s^{i} \mathbf{t}^{\mathbf{m}}\right)\right] z_{1}^{-j h} z_{2}^{-i} .
$$

The corresponding equalities for $T_{i} \otimes s^{b_{i}} \mathbf{t}^{\mathbf{m}}$ and $s^{i h} \mathbf{t}^{\mathbf{m}} K_{p}$ will follow since $s^{j h} \mathbf{t}^{\mathbf{r}} D_{p}$ acts as a derivation and $s^{i} \mathbf{t}^{\mathbf{m}} A_{i}^{\alpha}$ generate $\sum_{j \in \mathbb{Z}} \dot{\mathfrak{g}}_{j} \otimes s^{j} \mathbb{C}\left[t_{1}^{ \pm}, \ldots, t_{n}^{ \pm}\right] \oplus \mathcal{K}$.

Let us prove that (4.2) holds.

$$
\begin{aligned}
& \sum_{j, i \in \mathbb{Z}} \varphi\left(\left[s^{j h} \mathbf{t}^{\mathbf{r}} D_{p}, s^{i} \mathbf{t}^{\mathbf{m}} A_{i}^{\alpha}\right]\right) z_{1}^{-j h} z_{2}^{-i}= \\
& \sum_{j, i \in \mathbb{Z}} m_{p} \varphi\left(s^{i+j h} \mathbf{t}^{\mathbf{r}+\mathbf{m}} A_{i}^{\alpha}\right) z_{1}^{-j h} z_{2}^{-i}= \\
& \sum_{j \in \mathbb{Z}} \sum_{k=i+j h \in \mathbb{Z}} m_{p} \varphi\left(s^{k} \mathbf{t}^{\mathbf{r}+\mathbf{m}} A_{k}^{\alpha}\right) z_{1}^{-j h} z_{2}^{-k+j h}= \\
& m_{p} A^{\alpha}\left(z_{2}, \mathbf{r}+\mathbf{m}\right) \delta\left(\left(\frac{z_{2}}{z_{1}}\right)^{h}\right) .
\end{aligned}
$$

Next, we will show that the right hand side of (4.2) reduces to the same expression. In the following computations it will be convenient to denote $\varphi\left(s^{j h} D_{p}\right)=\frac{\partial}{\partial u_{p j}}$ by $\gamma_{p}(j)$, 
$\varphi\left(s^{-j h} D_{p}\right)=j v_{p j}$ by $\gamma_{p}(-j)$ and $\varphi\left(D_{p}\right)=q_{p} \frac{\partial}{\partial q_{p}}$ by $\gamma_{p}(0)$. Note that $\left[\gamma_{p}(j), K_{0}(z, \mathbf{m})\right]=$ $m_{p} z^{j h} K_{0}(z, \mathbf{m})$ for $j \in \mathbb{Z}$.

$$
\begin{aligned}
& \sum_{j, i \in \mathbb{Z}}\left[\varphi\left(s^{j h} \mathbf{t}^{\mathbf{r}} D_{p}\right), \varphi\left(A_{i}^{\alpha} \otimes s^{i} \mathbf{t}^{\mathbf{m}}\right)\right] z_{1}^{-j h} z_{2}^{-i}=\left[D_{p}\left(z_{1}, \mathbf{r}\right), A^{\alpha}\left(z_{2}, \mathbf{m}\right)\right]= \\
& {\left[:\left\{\sum_{j \in \mathbb{Z}} \gamma_{p}(j) z_{1}^{-j h}\right\} K_{0}\left(z_{1}, \mathbf{r}\right):, A^{\alpha}\left(z_{2}\right) K_{0}\left(z_{2}, \mathbf{m}\right)\right]=} \\
& A^{\alpha}\left(z_{2}\right)\left[:\left\{\sum_{j \in \mathbb{Z}} \gamma_{p}(j) z_{1}^{-j h}\right\} K_{0}\left(z_{1}, \mathbf{r}\right):, K_{0}\left(z_{2}, \mathbf{m}\right)\right]= \\
& A^{\alpha}\left(z_{2}\right)\left[\left\{\sum_{j<0} \gamma_{p}(j) z_{1}^{-j h}\right\} K_{0}\left(z_{1}, \mathbf{r}\right), K_{0}\left(z_{2}, \mathbf{m}\right)\right]+ \\
& A^{\alpha}\left(z_{2}\right)\left[K_{0}\left(z_{1}, \mathbf{r}\right)\left\{\sum_{j \geq 0} \gamma_{p}(j) z_{1}^{-j h}\right\}, K_{0}\left(z_{2}, \mathbf{m}\right)\right]= \\
& A^{\alpha}\left(z_{2}\right)\left[\sum_{j<0} \gamma_{p}(j) z_{1}^{-j h}, K_{0}\left(z_{2}, \mathbf{m}\right)\right] K_{0}\left(z_{1}, \mathbf{r}\right)+ \\
& A^{\alpha}\left(z_{2}\right) K_{0}\left(z_{1}, \mathbf{r}\right)\left[\sum_{j \geq 0} \gamma_{p}(j) z_{1}^{-j h}, K_{0}\left(z_{2}, \mathbf{m}\right)\right]= \\
& A^{\alpha}\left(z_{2}\right) \sum_{j<0} z_{1}^{-j h} z_{2}^{j h} m_{p} K_{0}\left(z_{2}, \mathbf{m}\right) K_{0}\left(z_{1}, \mathbf{r}\right)+ \\
& A^{\alpha}\left(z_{2}\right) K_{0}\left(z_{1}, \mathbf{r}\right) \sum_{j \geq 0} z_{1}^{-j h} z_{2}^{j h} m_{p} K_{0}\left(z_{2}, \mathbf{m}\right)= \\
& m_{p} A^{\alpha}\left(z_{2}\right) K_{0}\left(z_{1}, \mathbf{r}\right) K_{0}\left(z_{2}, \mathbf{m}\right) \delta\left(\left(\frac{z_{2}}{z_{1}}\right)^{h}\right)= \\
& m_{p} A^{\alpha}\left(z_{2}\right) K_{0}\left(z_{2}, \mathbf{r}\right) K_{0}\left(z_{2}, \mathbf{m}\right) \delta\left(\left(\frac{z_{2}}{z_{1}}\right)^{h}\right)= \\
& m_{p} A^{\alpha}\left(z_{2}, \mathbf{r}+\mathbf{m}\right) \delta\left(\left(\frac{z_{2}}{z_{1}}\right)^{h}\right) .
\end{aligned}
$$

From the above computation we can see that

$$
\left[D_{p}\left(z_{1}, \mathbf{r}\right), K_{0}\left(z_{2}, \mathbf{m}\right)\right]=m_{p} K_{0}\left(z_{2}, \mathbf{r}+\mathbf{m}\right) \delta\left(\left(\frac{z_{2}}{z_{1}}\right)^{h}\right) .
$$

To complete the proof of Theorem 5 we need to compute the commutators $\left[\varphi\left(s^{j h} \mathbf{t}^{\mathbf{r}} D_{a}\right), \varphi\left(s^{i h} \mathbf{t}^{\mathbf{m}} D_{b}\right)\right]$. We will check the following equality for the generating series:

$$
\sum_{j, i \in \mathbb{Z}} \varphi\left(\left[s^{j h} \mathbf{t}^{\mathbf{r}} D_{a}, s^{i h} \mathbf{t}^{\mathbf{m}} D_{b}\right]\right) z_{1}^{-j h} z_{2}^{-i h}=\sum_{j, i \in \mathbb{Z}}\left[\varphi\left(s^{j h} \mathbf{t}^{\mathbf{r}} D_{a}\right), \varphi\left(s^{i h} \mathbf{t}^{\mathbf{m}} D_{b}\right)\right] z_{1}^{-j h} z_{2}^{-i h} .
$$


To compute the left hand side we use multiplication in $\mathfrak{g}$ :

$$
\begin{aligned}
\sum_{j, i \in \mathbb{Z}} \varphi & \left(\left[s^{j h} \mathbf{t}^{\mathbf{r}} D_{a}, s^{i h} \mathbf{t}^{\mathbf{m}} D_{b}\right]\right) z_{1}^{-j h} z_{2}^{-i h}= \\
= & \sum_{j, i \in \mathbb{Z}} \varphi\left(m_{a} s^{(j+i) h} \mathbf{t}^{\mathbf{r}+\mathbf{m}} D_{b}-r_{b} s^{(j+i) h} \mathbf{t}^{\mathbf{r}+\mathbf{m}} D_{a}\right) z_{1}^{-j h} z_{2}^{-i h}- \\
& m_{a} r_{b} \varphi\left(s^{(j+i) h} \mathbf{t}^{\mathbf{r}+\mathbf{m}}\left\{j K_{0}+\sum_{p=1}^{n} r_{p} K_{p}\right\}\right) z_{1}^{-j h} z_{2}^{-i h}= \\
= & \sum_{j \in \mathbb{Z}} \sum_{k=j+i \in \mathbb{Z}}\left(m_{a} \varphi\left(s^{k h} \mathbf{t}^{\mathbf{r}+\mathbf{m}} D_{b}\right)-r_{b} \varphi\left(s^{k h} \mathbf{t}^{\mathbf{r}+\mathbf{m}} D_{a}\right)\right) z_{1}^{-j h} z_{2}^{-k h+j h}- \\
& \left.\sum_{j \in \mathbb{Z}} \sum_{k=j+i \in \mathbb{Z}} m_{a} r_{b} \varphi\left(s^{k h} \mathbf{t}^{\mathbf{r}+\mathbf{m}}\left\{j K_{0}+\sum_{p=1}^{n} r_{p} K_{p}\right\}\right)\right) z_{1}^{-j h} z_{2}^{-k h+j h}= \\
= & \left(m_{a} D_{b}\left(z_{2}, \mathbf{r}+\mathbf{m}\right)-r_{b} D_{a}\left(z_{2}, \mathbf{r}+\mathbf{m}\right)\right) \delta\left(\left(\frac{z_{2}}{z_{1}}\right)^{h}\right)- \\
& m_{a} r_{b} \sum_{p=1}^{n} r_{p} K_{p}\left(z_{2}, \mathbf{r}+\mathbf{m}\right) \delta\left(\left(\frac{z_{2}}{z_{1}}\right)^{h}\right)-m_{a} r_{b} K_{0}\left(z_{2}, \mathbf{r}+\mathbf{m}\right) D \delta\left(\left(\frac{z_{2}}{z_{1}}\right)^{h}\right) .
\end{aligned}
$$

Observe that

$$
\begin{gathered}
{\left[D_{a}(z, \mathbf{r}), \gamma_{b}(i)\right]=\left[:\left\{\sum_{j \in \mathbb{Z}} \gamma_{a}(j) z^{-j h}\right\} K_{0}(z, \mathbf{r}):, \gamma_{b}(i)\right]=} \\
{\left[\sum_{j<0} \gamma_{a}(j) z^{-j h} K_{0}(z, \mathbf{r}), \gamma_{b}(i)\right]+\left[K_{0}(z, \mathbf{r}) \sum_{j \geq 0} \gamma_{a}(j) z^{-j h}, \gamma_{b}(i)\right]=} \\
\sum_{j<0} \gamma_{a}(j) z^{-j h}\left[K_{0}(z, \mathbf{r}), \gamma_{b}(i)\right]+\left[K_{0}(z, \mathbf{r}), \gamma_{b}(i)\right] \sum_{j \geq 0} \gamma_{a}(j) z^{-j h}= \\
-r_{b} z^{i h} \sum_{j<0} \gamma_{a}(j) z^{-j h} K_{0}(z, \mathbf{r})-r_{b} z^{i h} K_{0}(z, \mathbf{r}) \sum_{j \geq 0} \gamma_{a}(j) z^{-j h}= \\
-r_{b} z^{i h} D_{a}(z, \mathbf{r}) .
\end{gathered}
$$

We use the last equality to compute the right hand side of (4.3):

$$
\begin{aligned}
& \sum_{j, i \in \mathbb{Z}}\left[\varphi\left(s^{j h} \mathbf{t}^{\mathbf{r}} D_{a}\right), \varphi\left(s^{i h} \mathbf{t}^{\mathbf{m}} D_{b}\right)\right] z_{1}^{-j h} z_{2}^{-i h}=\left[D_{a}\left(z_{1}, \mathbf{r}\right), D_{b}\left(z_{2}, \mathbf{m}\right)\right]= \\
& =\left[D_{a}\left(z_{1}, \mathbf{r}\right), \sum_{i<0} \gamma_{b}(i) z_{2}^{-i h} K_{0}\left(z_{2}, \mathbf{m}\right)\right]+\left[D_{a}\left(z_{1}, \mathbf{r}\right), K_{0}\left(z_{2}, \mathbf{m}\right) \sum_{i \geq 0} \gamma_{b}(i) z_{2}^{-i h}\right]=
\end{aligned}
$$




$$
\begin{aligned}
& =\sum_{i<0}\left[D_{a}\left(z_{1}, \mathbf{r}\right), \gamma_{b}(i)\right] z_{2}^{-i h} K_{0}\left(z_{2}, \mathbf{m}\right)+\sum_{i<0} \gamma_{b}(i) z_{2}^{-i h}\left[D_{a}\left(z_{1}, \mathbf{r}\right), K_{0}\left(z_{2}, \mathbf{m}\right)\right]+ \\
& {\left[D_{a}\left(z_{1}, \mathbf{r}\right), K_{0}\left(z_{2}, \mathbf{m}\right)\right] \sum_{i \geq 0} \gamma_{b}(i) z_{2}^{-i h}+K_{0}\left(z_{2}, \mathbf{m}\right) \sum_{i \geq 0}\left[D_{a}\left(z_{1}, \mathbf{r}\right), \gamma_{b}(i)\right] z_{2}^{-i h}=} \\
& =-r_{b} \sum_{i<0} D_{a}\left(z_{1}, \mathbf{r}\right) z_{1}^{i h} z_{2}^{-i h} K_{0}\left(z_{2}, \mathbf{m}\right)+\sum_{i<0} \gamma_{b}(i) z_{2}^{-i h} m_{a} K_{0}\left(z_{2}, \mathbf{r}+\mathbf{m}\right) \delta\left(\left(\frac{z_{2}}{z_{1}}\right)^{h}\right)+ \\
& m_{a} K_{0}\left(z_{2}, \mathbf{r}+\mathbf{m}\right) \delta\left(\left(\frac{z_{2}}{z_{1}}\right)^{h}\right) \sum_{i \geq 0} \gamma_{b}(i) z_{2}^{-i h}-r_{b} K_{0}\left(z_{2}, \mathbf{m}\right) \sum_{i \geq 0} D_{a}\left(z_{1}, \mathbf{r}\right) z_{1}^{i h} z_{2}^{-i h}= \\
& =m_{a} D_{b}\left(z_{2}, \mathbf{r}+\mathbf{m}\right) \delta\left(\left(\frac{z_{2}}{z_{1}}\right)^{h}\right)- \\
& r_{b} \sum_{i<0}\left(\frac{z_{1}}{z_{2}}\right)^{i h} \sum_{j<0} \gamma_{a}(j) z_{1}^{-j h} K_{0}\left(z_{1}, \mathbf{r}\right) K_{0}\left(z_{2}, \mathbf{m}\right)- \\
& r_{b} \sum_{i<0}\left(\frac{z_{1}}{z_{2}}\right)^{i h} K_{0}\left(z_{1}, \mathbf{r}\right) \sum_{j \geq 0} \gamma_{a}(j) z_{1}^{-j h} K_{0}\left(z_{2}, \mathbf{m}\right)- \\
& r_{b} K_{0}\left(z_{2}, \mathbf{m}\right) \sum_{i \geq 0} \sum_{j<0} \gamma_{a}(j) z_{1}^{-j h} K_{0}\left(z_{1}, \mathbf{r}\right)\left(\frac{z_{1}}{z_{2}}\right)^{i h}- \\
& r_{b} K_{0}\left(z_{2}, \mathbf{m}\right) \sum_{i \geq 0} K_{0}\left(z_{1}, \mathbf{r}\right)\left(\frac{z_{1}}{z_{2}}\right)^{i h} \sum_{j \geq 0} \gamma_{a}(j) z_{1}^{-j h}= \\
& =m_{a} D_{b}\left(z_{2}, \mathbf{r}+\mathbf{m}\right) \delta\left(\left(\frac{z_{2}}{z_{1}}\right)^{h}\right)- \\
& r_{b} \sum_{i<0} \sum_{j<0}\left(\frac{z_{1}}{z_{2}}\right)^{i h} z_{1}^{-j h} \gamma_{a}(j) K_{0}\left(z_{1}, \mathbf{r}\right) K_{0}\left(z_{2}, \mathbf{m}\right)- \\
& r_{b} \sum_{i<0} \sum_{j \geq 0}\left(\frac{z_{1}}{z_{2}}\right)^{i h} z_{1}^{-j h} K_{0}\left(z_{1}, \mathbf{r}\right)\left[\gamma_{a}(j), K_{0}\left(z_{2}, \mathbf{m}\right)\right]- \\
& r_{b} \sum_{i<0} \sum_{j \geq 0}\left(\frac{z_{1}}{z_{2}}\right)^{i h} z_{1}^{-j h} K_{0}\left(z_{1}, \mathbf{r}\right) K_{0}\left(z_{2}, \mathbf{m}\right) \gamma_{a}(j)- \\
& r_{b} \sum_{i \geq 0} \sum_{j<0} z_{1}^{-j h}\left(\frac{z_{1}}{z_{2}}\right)^{i h}\left[K_{0}\left(z_{2}, \mathbf{m}\right), \gamma_{a}(j)\right] K_{0}\left(z_{1}, \mathbf{r}\right)- \\
& r_{b} \sum_{i \geq 0} \sum_{j<0} z_{1}^{-j h}\left(\frac{z_{1}}{z_{2}}\right)^{i h} \gamma_{a}(j) K_{0}\left(z_{2}, \mathbf{m}\right) K_{0}\left(z_{1}, \mathbf{r}\right)- \\
& r_{b} \sum_{i \geq 0} \sum_{j \geq 0}\left(\frac{z_{1}}{z_{2}}\right)^{i h} z_{1}^{-j h} K_{0}\left(z_{2}, \mathbf{m}\right) K_{0}\left(z_{1}, \mathbf{r}\right) \gamma_{a}(j)=
\end{aligned}
$$




$$
\begin{aligned}
& =m_{a} D_{b}\left(z_{2}, \mathbf{r}+\mathbf{m}\right) \delta\left(\left(\frac{z_{2}}{z_{1}}\right)^{h}\right)- \\
& r_{b} \delta\left(\left(\frac{z_{2}}{z_{1}}\right)^{h}\right):\left\{\sum_{j \in \mathbb{Z}} \gamma_{a}(j) z_{2}^{-j h}\right\} K_{0}\left(z_{2}, \mathbf{r}\right) K_{0}\left(z_{2}, \mathbf{m}\right):- \\
& m_{a} r_{b} \sum_{i>0} \sum_{j \geq 0}\left(\frac{z_{2}}{z_{1}}\right)^{(i+j) h} K_{0}\left(z_{1}, \mathbf{r}\right) K_{0}\left(z_{2}, \mathbf{m}\right)+ \\
& m_{a} r_{b} \sum_{i \leq 0} \sum_{j<0}\left(\frac{z_{2}}{z_{1}}\right)^{(i+j) h} K_{0}\left(z_{1}, \mathbf{r}\right) K_{0}\left(z_{2}, \mathbf{m}\right)= \\
& =m_{a} D_{b}\left(z_{2}, \mathbf{r}+\mathbf{m}\right) \delta\left(\left(\frac{z_{2}}{z_{1}}\right)^{h}\right)-r_{b} D_{a}\left(z_{2}, \mathbf{r}+\mathbf{m}\right) \delta\left(\left(\frac{z_{2}}{z_{1}}\right)^{h}\right)- \\
& m_{a} r_{b} \sum_{k=i+j>0} \sum_{j=0}^{k-1}\left(\frac{z_{2}}{z_{1}}\right)^{k h} K_{0}\left(z_{1}, \mathbf{r}\right) K_{0}\left(z_{2}, \mathbf{m}\right)+ \\
& m_{a} r_{b} \sum_{k=i+j<0} \sum_{j=k}^{-1}\left(\frac{z_{2}}{z_{1}}\right)^{k h} K_{0}\left(z_{1}, \mathbf{r}\right) K_{0}\left(z_{2}, \mathbf{m}\right)= \\
& =\left(m_{a} D_{b}\left(z_{2}, \mathbf{r}+\mathbf{m}\right)-r_{b} D_{a}\left(z_{2}, \mathbf{r}+\mathbf{m}\right)\right) \delta\left(\left(\frac{z_{2}}{z_{1}}\right)^{h}\right)- \\
& m_{a} r_{b} \sum_{k \in \mathbb{Z}} k\left(\frac{z_{2}}{z_{1}}\right)^{k h} K_{0}\left(z_{1}, \mathbf{r}\right) K_{0}\left(z_{2}, \mathbf{m}\right)= \\
& =\left(m_{a} D_{b}\left(z_{2}, \mathbf{r}+\mathbf{m}\right)-r_{b} D_{a}\left(z_{2}, \mathbf{r}+\mathbf{m}\right)\right) \delta\left(\left(\frac{z_{2}}{z_{1}}\right)^{h}\right)- \\
& m_{a} r_{b} K_{0}\left(z_{1}, \mathbf{r}\right) K_{0}\left(z_{2}, \mathbf{m}\right) D \delta\left(\left(\frac{z_{2}}{z_{1}}\right)^{h}\right)= \\
& =\left(m_{a} D_{b}\left(z_{2}, \mathbf{r}+\mathbf{m}\right)-r_{b} D_{a}\left(z_{2}, \mathbf{r}+\mathbf{m}\right)\right) \delta\left(\left(\frac{z_{2}}{z_{1}}\right)^{h}\right)- \\
& \frac{1}{h} m_{a} r_{b} D_{z_{2}}\left(K_{0}\left(z_{2}, \mathbf{r}\right)\right) K_{0}\left(z_{2}, \mathbf{m}\right) \delta\left(\left(\frac{z_{2}}{z_{1}}\right)^{h}\right)- \\
& m_{a} r_{b} K_{0}\left(z_{2}, \mathbf{r}\right) K_{0}\left(z_{2}, \mathbf{m}\right) D \delta\left(\left(\frac{z_{2}}{z_{1}}\right)^{h}\right)= \\
& =\left(m_{a} D_{b}\left(z_{2}, \mathbf{r}+\mathbf{m}\right)-r_{b} D_{a}\left(z_{2}, \mathbf{r}+\mathbf{m}\right)\right) \delta\left(\left(\frac{z_{2}}{z_{1}}\right)^{h}\right)- \\
& m_{a} r_{b} \sum_{p=1}^{n} r_{p} K_{p}\left(z_{2}, \mathbf{r}+\mathbf{m}\right) \delta\left(\left(\frac{z_{2}}{z_{1}}\right)^{h}\right)- \\
& m_{a} r_{b} K_{0}\left(z_{2}, \mathbf{r}+\mathbf{m}\right) D \delta\left(\left(\frac{z_{2}}{z_{1}}\right)^{h}\right) .
\end{aligned}
$$

This establishes (4.3) and completes the proof of Theorem 5. 


\section{Sugawara operators.}

In the representation we constructed the operators from $\mathcal{D}_{+}$act as derivations on the algebra $\hat{\mathfrak{g}}=\dot{\mathfrak{g}} \otimes \mathbb{C}\left[t_{0}^{ \pm}, t_{1}^{ \pm}, \ldots, t_{n}^{ \pm}\right] \oplus \mathcal{K}$. It is possible to extend this action on $\mathcal{D}$, that is to represent $t_{0}^{j} \mathbf{t}^{\mathbf{r}} D_{0}$ by operators on $F$. For the affine case these are Sugawara operators.

We will work with the toroidal algebra realized as $\sum_{j \in \mathbb{Z}} \dot{\mathfrak{g}}_{j} \otimes s^{j} \mathbb{C}\left[t_{1}^{ \pm}, \ldots, t_{n}^{ \pm}\right] \oplus \mathcal{K}$. For this realization

$$
\psi\left(t_{0}^{j} \mathbf{t}^{\mathbf{r}} D_{0}\right)=\frac{1}{h} s^{j h} \mathbf{t}^{\mathbf{r}} D_{s}-\frac{1}{h} \nu^{-1}(\rho) \otimes s^{j h} \mathbf{t}^{\mathbf{r}},
$$

where $\rho \in \dot{\mathfrak{h}}$ with $\left(\rho \mid \alpha_{i}\right)=1$ for every simple root $\alpha_{i} \in \dot{\Delta}$ and $D_{s}=s \frac{d}{d s}$.

Since for $\alpha \in \Delta$ the elements $A^{\alpha} \otimes t_{0}^{i} \mathbf{t}^{\mathbf{m}}$ generate $\hat{\mathfrak{g}}$ then we have to check that

$$
\psi\left(\left[t_{0}^{j} \mathbf{t}^{\mathbf{r}} D_{0}, A^{\alpha} \otimes t_{0}^{i} \mathbf{t}^{\mathbf{m}}\right]\right)=\left[\psi\left(t_{0}^{j} \mathbf{t}^{\mathbf{r}} D_{0}\right), \psi\left(A^{\alpha} \otimes t_{0}^{i} \mathbf{t}^{\mathbf{m}}\right)\right] .
$$

Note that $A^{\alpha} \in \dot{\mathfrak{g}}_{\mathrm{ht}(\alpha)}$ and $(\rho \mid \alpha)=\operatorname{ht}(\alpha)$.

We have

$$
\psi\left(\left[t_{0}^{j} \mathbf{t}^{\mathbf{r}} D_{0}, A^{\alpha} \otimes t_{0}^{i} \mathbf{t}^{\mathbf{m}}\right]\right)=i \psi\left(A^{\alpha} \otimes t_{0}^{i+j} \mathbf{t}^{\mathbf{r}+\mathbf{m}}\right)=i A^{\alpha} \otimes s^{\mathrm{ht}(\alpha)+h(i+j)} \mathbf{t}^{\mathbf{r}+\mathbf{m}},
$$

while

$$
\begin{gathered}
{\left[\psi\left(t_{0}^{j} \mathbf{t}^{\mathbf{r}} D_{0}\right), \psi\left(A^{\alpha} \otimes t_{0}^{i} \mathbf{t}^{\mathbf{m}}\right)\right]=} \\
{\left[\frac{1}{h} s^{j h} \mathbf{t}^{\mathbf{r}} D_{s}-\frac{1}{h} \nu^{-1}(\rho) \otimes s^{j h} \mathbf{t}^{\mathbf{r}}, A^{\alpha} \otimes s^{\operatorname{ht}(\alpha)+i h} \mathbf{t}^{\mathbf{m}}\right]=} \\
\frac{1}{h}(\operatorname{ht}(\alpha)+i h-(\rho \mid \alpha)) A^{\alpha} \otimes s^{\operatorname{ht}(\alpha)+h(i+j)} \mathbf{t}^{\mathbf{r}+\mathbf{m}}= \\
i A^{\alpha} \otimes s^{\operatorname{ht}(\alpha)+h(i+j)} \mathbf{t}^{\mathbf{r}+\mathbf{m}} .
\end{gathered}
$$

Thus it is sufficient to construct operators on $F$ corresponding to $s^{j h} \mathbf{t}^{\mathbf{r}} D_{s}$.

It will be convenient to denote in this section $\varphi\left(T_{i} \otimes s^{b_{i}}\right)$ by $\tau(i), \varphi\left(s^{j h} D_{p}\right)$ by $\gamma_{p}(j)$ and $\varphi\left(s^{j h} K_{p}\right)$ by $\kappa_{p}(j)$.

In these notations

$$
\begin{aligned}
K_{0}(z, \mathbf{r}) & =\mathbf{q}^{\mathbf{r}} \exp \left(\sum_{p=1}^{n} r_{p} \sum_{j \geq 1} \frac{\kappa_{p}(-j)}{j} z^{j h}\right) \exp \left(-\sum_{p=1}^{n} r_{p} \sum_{j \geq 1} \frac{\kappa_{p}(j)}{j} z^{-j h}\right) \quad \text { and } \\
A^{\alpha}(z) & =\Lambda_{0}\left(A_{0}^{\alpha} \otimes s^{0}\right) \exp \left(\sum_{i \geq 1} \lambda_{i}^{\alpha} \frac{\tau(1-i)}{b_{i}} z^{b_{i}}\right) \exp \left(-\sum_{i \geq 1} \lambda_{1-i}^{\alpha} \frac{\tau(i)}{b_{i}} z^{-b_{i}}\right) .
\end{aligned}
$$


Nontrivial commutators in the principal Heisenberg subalgebra are

$$
[\tau(i), \tau(1-i)]=b_{i} \text { and }\left[\gamma_{p}(j), \kappa_{p}(-j)\right]=j
$$

The action of the Heisenberg subalgebra on the vertex operators is determined by

$$
\begin{gathered}
{\left[\tau(i), A^{\alpha}(z)\right]=\lambda_{i}^{\alpha} z^{b_{i}} A^{\alpha}(z),} \\
{\left[\gamma_{p}(j), K_{0}(z, \mathbf{r})\right]=r_{p} z^{j h} K_{0}(z, \mathbf{r}) .}
\end{gathered}
$$

Consider the following operators on the Fock space $F$ :

$$
\begin{gathered}
L_{\tau}(j)=\frac{1}{2} \sum_{i \in \mathbb{Z}}: \tau(1-i) \tau(i+j \ell):, \\
L_{\gamma \kappa}(j)=\sum_{p=1}^{n} \sum_{i \in \mathbb{Z}}: \gamma_{p}(-i) \kappa_{p}(i+j): .
\end{gathered}
$$

These are the analogues of the Sugawara operators.

Construct the formal generating series for these:

$$
D_{s}(z)=-\sum_{j \in \mathbb{Z}}\left(L_{\tau}(j)+h L_{\gamma \kappa}(j)\right) z^{-j h} .
$$

Proposition 8. Formula (3.5) together with

$$
\sum_{i \in \mathbb{Z}} \varphi\left(s^{i h} \mathbf{t}^{\mathbf{r}} D_{s}\right) z^{-i h}=D_{s}(z, \mathbf{r})=: D_{s}(z) K_{0}(z, \mathbf{r}):
$$

defines the representation of the Lie algebra $\mathcal{D}$ on the space

$$
\varphi(\hat{\mathfrak{g}})=\varphi\left(\sum_{j \in \mathbb{Z}} \dot{\mathfrak{g}}_{j} \otimes s^{j} \mathbb{C}\left[t_{1}^{ \pm}, \ldots, t_{n}^{ \pm}\right] \oplus \mathcal{K}\right) .
$$

Proof. We have an action of $\mathcal{D}$ on $\hat{\mathfrak{g}}$ which is a unique extension of its natural action on $\tilde{\mathfrak{g}}$. Thus we need to prove that $\varphi([D, B])=[\varphi(D), \varphi(B)]$ for every $D \in \mathcal{D}$ and $B \in \hat{\mathfrak{g}}$. For subalgebra $\mathcal{D}_{+}$this was proved in the course of Theorem 4 , hence we need to consider only $D=s^{i h} \mathbf{t}^{\mathbf{r}} D_{s}$. Since $s^{i h} \mathbf{t}^{\mathbf{r}} D_{s}$ act on $\hat{\mathfrak{g}}$ as derivations and $\hat{\mathfrak{g}}$ is generated as an algebra by elements $A_{j}^{\alpha} \otimes s^{j} \mathbf{t}^{\mathbf{m}}$ then it is sufficient to prove that

$$
\varphi\left(\left[s^{i h} \mathbf{t}^{\mathbf{r}} D_{s}, A_{j}^{\alpha} \otimes s^{j} \mathbf{t}^{\mathbf{m}}\right]\right)=\left[\varphi\left(s^{i h} \mathbf{t}^{\mathbf{r}} D_{s}\right), \varphi\left(A_{j}^{\alpha} \otimes s^{j} \mathbf{t}^{\mathbf{m}}\right)\right] .
$$


Again we shall replace this with an equivalent identity for the corresponding series:

$$
\varphi\left(\left[\sum_{i \in \mathbb{Z}} s^{i h} \mathbf{t}^{\mathbf{r}} D_{s} z_{1}^{-i h}, \sum_{j \in \mathbb{Z}} A_{j}^{\alpha} \otimes s^{j} \mathbf{t}^{\mathbf{m}} z_{2}^{-j}\right]\right)=\left[\sum_{i \in \mathbb{Z}} \varphi\left(s^{i h} \mathbf{t}^{\mathbf{r}} D_{s}\right) z_{1}^{-i h}, \sum_{j \in \mathbb{Z}} \varphi\left(A_{j}^{\alpha} \otimes s^{j} \mathbf{t}^{\mathbf{m}}\right) z_{2}^{-j}\right] .
$$

We have

$$
\begin{aligned}
& \varphi\left(\left[\sum_{i \in \mathbb{Z}} s^{i h} \mathbf{t}^{\mathbf{r}} D_{s} z_{1}^{-i h}, \sum_{j \in \mathbb{Z}} A_{j}^{\alpha} \otimes s^{j} \mathbf{t}^{\mathbf{m}} z_{2}^{-j}\right]\right)= \\
&=\sum_{i \in \mathbb{Z}} \sum_{j \in \mathbb{Z}} j \varphi\left(A_{j}^{\alpha} \otimes s^{j+i h} \mathbf{t}^{\mathbf{r}+\mathbf{m}}\right) z_{1}^{-i h} z_{2}^{-j}= \\
&=\sum_{i \in \mathbb{Z}} \sum_{k=j+i h \in \mathbb{Z}}(k-i h) \varphi\left(A_{k}^{\alpha} \otimes s^{k} \mathbf{t}^{\mathbf{r}+\mathbf{m}}\right) z_{1}^{-i h} z_{2}^{-k+i h}= \\
&= \sum_{k \in \mathbb{Z}} k \varphi\left(A_{k}^{\alpha} \otimes s^{k} \mathbf{t}^{\mathbf{r}+\mathbf{m}}\right) z_{2}^{-k} \delta\left(\left(\frac{z_{2}}{z_{1}}\right)^{h}\right)- \\
& h \sum_{k \in \mathbb{Z}} \varphi\left(A_{k}^{\alpha} \otimes s^{k} \mathbf{t}^{\mathbf{r}+\mathbf{m}}\right) z_{2}^{-k} D \delta\left(\left(\frac{z_{2}}{z_{1}}\right)^{h}\right)= \\
&=-\left(D_{z_{2}} A^{\alpha}\left(z_{2}, \mathbf{r}+\mathbf{m}\right)\right) \delta\left(\left(\frac{z_{2}}{z_{1}}\right)^{h}\right)- \\
& A^{\alpha}\left(z_{2}, \mathbf{r}+\mathbf{m}\right)\left(D_{z_{2}} \delta\left(\left(\frac{z_{2}}{z_{1}}\right)^{h}\right)\right)= \\
&=-D_{z_{2}}\left(A^{\alpha}\left(z_{2}, \mathbf{r}+\mathbf{m}\right) \delta\left(\left(\frac{z_{2}}{z_{1}}\right)^{h}\right)\right)
\end{aligned}
$$

where $D_{z_{2}}=z_{2} \frac{\partial}{\partial z_{2}}$.

In order to compute the right hand side of (5.1), we will need two lemmas.

\section{Lemma 9.}

$$
\begin{gathered}
{\left[L_{\tau}(j), A^{\alpha}(z)\right]=z^{j h}\left(D_{z}+j h\right) A^{\alpha}(z),} \\
{\left[L_{\tau}\left(z_{1}\right), A^{\alpha}\left(z_{2}\right)\right]=D_{z_{2}}\left(A^{\alpha}\left(z_{2}\right) \delta\left(\left(\frac{z_{2}}{z_{1}}\right)^{h}\right)\right) .}
\end{gathered}
$$

Proof. We will prove (i) assuming that $j \geq 0$. The case $j<0$ is analogous.

$$
\begin{gathered}
{\left[L_{\tau}(j), A^{\alpha}(z)\right]=\frac{1}{2}\left[\sum_{i \in \mathbb{Z}}: \tau(1-i) \tau(i+j \ell): A^{\alpha}(z)\right]=} \\
=\frac{1}{2}\left[\sum_{i>0} \tau(1-i) \tau(i+j \ell), A^{\alpha}(z)\right]+\frac{1}{2}\left[\sum_{i \leq 0} \tau(i+j \ell) \tau(1-i), A^{\alpha}(z)\right]=
\end{gathered}
$$




$$
\begin{aligned}
& =\frac{1}{2} \sum_{i>0} \tau(1-i)\left[\tau(i+j \ell), A^{\alpha}(z)\right]+\frac{1}{2} \sum_{i>0}\left[\tau(1-i), A^{\alpha}(z)\right] \tau(i+j \ell)+ \\
& \frac{1}{2} \sum_{i \leq 0} \tau(i+j \ell)\left[\tau(1-i), A^{\alpha}(z)\right]+\frac{1}{2} \sum_{i \leq 0}\left[\tau(i+j \ell), A^{\alpha}(z)\right] \tau(1-i)= \\
& =\frac{1}{2} \sum_{i>0} \tau(1-i) \lambda_{i}^{\alpha} z^{b_{i}+j h} A^{\alpha}(z)+\frac{1}{2} \sum_{i>0} \lambda_{1-i}^{\alpha} z^{-b_{i}} A^{\alpha}(z) \tau(i+j \ell)+ \\
& \frac{1}{2} \sum_{i \leq 0} \tau(i+j \ell) \lambda_{1-i}^{\alpha} z^{-b_{i}} A^{\alpha}(z)+\frac{1}{2} \sum_{i \leq 0} \lambda_{i}^{\alpha} z^{b_{i}+j h} A^{\alpha}(z) \tau(1-i)= \\
& =\frac{1}{2} \sum_{i>0} \tau(1-i) \lambda_{i}^{\alpha} z^{b_{i}+j h} A^{\alpha}(z)+\frac{1}{2} \sum_{i>0} \lambda_{1-i}^{\alpha} z^{-b_{i}} A^{\alpha}(z) \tau(i+j \ell)+ \\
& \frac{1}{2} \sum_{i \leq-j \ell} \tau(i+j \ell) \lambda_{1-i}^{\alpha} z^{-b_{i}} A^{\alpha}(z)+\frac{1}{2} \sum_{-j \ell<i \leq 0} \lambda_{1-i}^{\alpha} z^{-b_{i}}\left[\tau(i+j \ell), A^{\alpha}(z)\right]+ \\
& \frac{1}{2} \sum_{-j \ell<i \leq 0} \lambda_{1-i}^{\alpha} z^{-b_{i}} A^{\alpha}(z) \tau(i+j \ell)+\frac{1}{2} \sum_{i \leq 0} \lambda_{i}^{\alpha} z^{b_{i}+j h} A^{\alpha}(z) \tau(1-i)= \\
& =\frac{1}{2} \sum_{k=i>0} \tau(1-k) \lambda_{k}^{\alpha} z^{b_{k}+j h} A^{\alpha}(z)+\frac{1}{2} \sum_{k=i+j \ell>j \ell} \lambda_{1-k}^{\alpha} z^{-b_{k}+j h} A^{\alpha}(z) \tau(k)+ \\
& \frac{1}{2} \sum_{k=1-i-j \ell>0} \lambda_{k}^{\alpha} z^{b_{k}+j h} \tau(1-k) A^{\alpha}(z)+\frac{1}{2} \sum_{-j \ell<i \leq 0} \lambda_{1-i}^{\alpha} z^{-b_{i}} \lambda_{i}^{\alpha} z^{b_{i}+j h} A^{\alpha}(z)+ \\
& \frac{1}{2} \sum_{0<k=i+j \ell \leq j \ell} \lambda_{1-k}^{\alpha} z^{-b_{k}+j h} A^{\alpha}(z) \tau(k)+\frac{1}{2} \sum_{k=1-i>0} \lambda_{1-k}^{\alpha} z^{-b_{k}+j h} A^{\alpha}(z) \tau(k)= \\
& =z^{j h} D_{z} A^{\alpha}(z)+\frac{j}{2} z^{j h}\left(\sum_{k=1}^{\ell} \lambda_{k}^{\alpha} \lambda_{1-k}^{\alpha}\right) A^{\alpha}(z)= \\
& =z^{j h}\left(D_{z}+h j\right) A^{\alpha}(z) \text {. }
\end{aligned}
$$

At the last step we used the equality

$$
\begin{gathered}
\frac{1}{2} \sum_{k=1}^{\ell} \lambda_{k}^{\alpha} \lambda_{1-k}^{\alpha}=\frac{1}{2} \sum_{k=1}^{\ell}\left(T_{k} \mid \nu^{-1}(\alpha)\right)\left(T_{1-k} \mid \nu^{-1}(\alpha)\right)= \\
\frac{h}{2}\left(\nu^{-1}(\alpha) \mid \nu^{-1}(\alpha)\right)=\frac{h}{2}(\alpha \mid \alpha)=h,
\end{gathered}
$$

which follows from the fact that $\left\{\frac{1}{h} T_{1-k}\right\}$ is the dual basis for $\left\{T_{k}\right\}$. 
Part (ii) is an immediate consequence of (i):

$$
\begin{gathered}
{\left[L_{\tau}\left(z_{1}\right), A^{\alpha}\left(z_{2}\right)\right]=\sum_{j \in \mathbb{Z}} z_{1}^{-j h}\left[L_{\tau}(j), A^{\alpha}\left(z_{2}\right)\right]=} \\
\sum_{j \in \mathbb{Z}} z_{1}^{-j h} z_{2}^{j h}\left(D_{z_{2}}+h j\right) A^{\alpha}\left(z_{2}\right)=\left(D_{z_{2}} A^{\alpha}\left(z_{2}\right)\right) \delta\left(\left(\frac{z_{2}}{z_{1}}\right)^{h}\right)+A^{\alpha}\left(z_{2}\right)\left(D_{z_{2}} \delta\left(\left(\frac{z_{2}}{z_{1}}\right)^{h}\right)\right)= \\
D_{z_{2}}\left(A^{\alpha}\left(z_{2}\right) \delta\left(\left(\frac{z_{2}}{z_{1}}\right)^{h}\right)\right) .
\end{gathered}
$$

\section{Lemma 10.}

$$
\left[: L_{\gamma \kappa}\left(z_{1}\right) K_{0}\left(z_{1}, \mathbf{r}\right):, K_{0}\left(z_{2}, \mathbf{m}\right)\right]=\frac{1}{h} \delta\left(\left(\frac{z_{2}}{z_{1}}\right)^{h}\right) D_{z_{2}}\left(K_{0}\left(z_{2}, \mathbf{m}\right)\right) K_{0}\left(z_{1}, \mathbf{r}\right) .
$$

Proof.

$$
\begin{gathered}
{\left[: L_{\gamma \kappa}\left(z_{1}\right) K_{0}\left(z_{1}, \mathbf{r}\right):, K_{0}\left(z_{2}, \mathbf{m}\right)\right]=} \\
=\sum_{j \in \mathbb{Z}} z_{1}^{-j h} \sum_{p=1}^{n} \sum_{i>0}\left[\gamma_{p}(-i) \kappa_{p}(i+j) K_{0}\left(z_{1}, \mathbf{r}\right), K_{0}\left(z_{2}, \mathbf{m}\right)\right]+ \\
\sum_{j \in \mathbb{Z}} z_{1}^{-j h} \sum_{p=1}^{n} \sum_{i \leq 0}\left[\kappa_{p}(i+j) K_{0}\left(z_{1}, \mathbf{r}\right) \gamma_{p}(-i), K_{0}\left(z_{2}, \mathbf{m}\right)\right]= \\
=\sum_{j \in \mathbb{Z}} z_{1}^{-j h} \sum_{p=1}^{n} \sum_{i>0}\left[\gamma_{p}(-i), K_{0}\left(z_{2}, \mathbf{m}\right)\right] \kappa_{p}(i+j) K_{0}\left(z_{1}, \mathbf{r}\right)+ \\
\sum_{j \in \mathbb{Z}} z_{1}^{-j h} \sum_{p=1}^{n} \sum_{i \leq 0} \kappa_{p}(i+j) K_{0}\left(z_{1}, \mathbf{r}\right)\left[\gamma_{p}(-i), K_{0}\left(z_{2}, \mathbf{m}\right)\right]= \\
=\sum_{j \in \mathbb{Z}} z_{1}^{-j h} \sum_{p=1}^{n} m_{p} \sum_{i>0} z_{2}^{-i h} K_{0}\left(z_{2}, \mathbf{m}\right) \kappa_{p}(i+j) K_{0}\left(z_{1}, \mathbf{r}\right)+ \\
\sum_{j \in \mathbb{Z}} z_{1}^{-j h} \sum_{p=1}^{n} m_{p} \sum_{i \leq 0} \kappa_{p}(i+j) K_{0}\left(z_{1}, \mathbf{r}\right) z_{2}^{-i h} K_{0}\left(z_{2}, \mathbf{m}\right)= \\
=\sum_{j \in \mathbb{Z}} \sum_{k=i+j \in \mathbb{Z}} \sum_{p=1}^{n} m_{p} z_{1}^{-j h} z_{2}^{-k h+j h} \kappa_{p}(k) K_{0}\left(z_{2}, \mathbf{m}\right) K_{0}\left(z_{1}, \mathbf{r}\right)= \\
=\frac{1}{h} \delta\left(\left(\frac{z_{2}}{z_{1}}\right)^{h}\right) D_{z_{2}}\left(K_{0}\left(z_{2}, \mathbf{m}\right)\right) K_{0}\left(z_{1}, \mathbf{r}\right) .
\end{gathered}
$$


Using these two lemmas we can handle the right hand side of (5.1).

$$
\begin{aligned}
& {\left[\sum_{i \in \mathbb{Z}} \varphi\left(s^{i h} \mathbf{t}^{\mathbf{r}} D_{s}\right) z_{1}^{-i h}, \sum_{j \in \mathbb{Z}} \varphi\left(A_{j}^{\alpha} \otimes s^{j} \mathbf{t}^{\mathbf{m}}\right) z_{2}^{-j}\right]=\left[D_{s}\left(z_{1}, \mathbf{r}\right), A^{\alpha}\left(z_{2}, \mathbf{m}\right)\right]=} \\
&=\left[: D_{s}\left(z_{1}\right) K_{0}\left(z_{1}, \mathbf{r}\right):, A^{\alpha}\left(z_{2}\right) K_{0}\left(z_{2}, \mathbf{m}\right)\right]= \\
&=-\left[L_{\tau}\left(z_{1}\right) K_{0}\left(z_{1}, \mathbf{r}\right), A^{\alpha}\left(z_{2}\right) K_{0}\left(z_{2}, \mathbf{m}\right)\right]- \\
&-h\left[: L_{\gamma \kappa}\left(z_{1}\right) K_{0}\left(z_{1}, \mathbf{r}\right):, A^{\alpha}\left(z_{2}\right) K_{0}\left(z_{2}, \mathbf{m}\right)\right]= \\
&-\left[L_{\tau}\left(z_{1}\right), A^{\alpha}\left(z_{2}\right)\right] K_{0}\left(z_{1}, \mathbf{r}\right) K_{0}\left(z_{2}, \mathbf{m}\right)- \\
& h A^{\alpha}\left(z_{2}\right)\left[: L_{\gamma \kappa}\left(z_{1}\right) K_{0}\left(z_{1}, \mathbf{r}\right):, K_{0}\left(z_{2}, \mathbf{m}\right)\right]= \\
&=-D_{z_{2}}\left(A^{\alpha}\left(z_{2}\right) \delta\left(\left(\frac{z_{2}}{z_{1}}\right)^{h}\right)\right) K_{0}\left(z_{2}, \mathbf{m}\right) K_{0}\left(z_{1}, \mathbf{r}\right)- \\
& A^{\alpha}\left(z_{2}\right) \delta\left(\left(\frac{z_{2}}{z_{1}}\right)^{h}\right) D_{z_{2}}\left(K_{0}\left(z_{2}, \mathbf{m}\right)\right) K_{0}\left(z_{1}, \mathbf{r}\right)= \\
&=-D_{z_{2}}\left(A^{\alpha}\left(z_{2}\right) \delta\left(\left(\frac{z_{2}}{z_{1}}\right)^{h}\right) K_{0}\left(z_{2}, \mathbf{m}\right) K_{0}\left(z_{1}, \mathbf{r}\right)\right)= \\
&=-D_{z_{2}}\left(A^{\alpha}\left(z_{2}\right) \delta\left(\left(\frac{z_{2}}{z_{1}}\right)^{h}\right) K_{0}\left(z_{2}, \mathbf{m}\right) K_{0}\left(z_{2}, \mathbf{r}\right)\right)= \\
&=-D_{z_{2}}\left(A^{\alpha}\left(z_{2}, \mathbf{r}+\mathbf{m}\right) \delta\left(\left(\frac{z_{2}}{z_{1}}\right)^{h}\right)\right) .
\end{aligned}
$$




\section{References}

[BM] G.M. Benkart, R.V. Moody, Derivations, central extensions, and affine Lie algebras, Algebras Groups Geom., 3 (1986), 456-492.

[BC] S. Berman, B. Cox, Enveloping algebras and representations of toroidal Lie algebras, Pacific J.Math., 165 (1994), 239-267.

[Co1] H.S.M. Coxeter, Regular polytopes, Methuen, London, 1948.

[Co2] H.S.M. Coxeter, Regular complex polytopes, Cambridge University Press, 1974.

[EM] S. Eswara Rao, R.V. Moody, Vertex representations for $n$-toroidal Lie algebras and a generalization of the Virasoro algebra, Comm. Math. Phys. 159 (1994), 239-264.

[FLM] I. Frenkel, J. Lepowsky, A. Meurman, Vertex operator algebras and the Monster, Academic Press, Boston, 1989.

[IKUX] T. Inami, H. Kanno, T. Ueno, C.-S. Xiong, Two-toroidal Lie algebra as current algebra of four-dimensional Kähler WZW model, hepth/9610187.

[Kac] V.G. Kac, Infinite-dimensional Lie algebras, 3rd ed., Cambridge University Press, 1990.

[KKLW] V.G. Kac, D.A. Kazhdan, J. Lepowsky, R.L. Wilson, Realization of the basic representation of the Euclidean Lie algebras, Adv.Math., 42 (1981), 83-112.

[Kas] C. Kassel, Kähler differentials and coverings of complex simple Lie algebras extended over a commutative algebra, J.Pure Appl.Algebra 34 (1985), 265-275.

[Kos] B. Kostant, The principal three-dimensional subgroup and the Betti numbers of a complex simple Lie group, Amer.J.Math. 81 (1959), 973-1032.

[MEY] R.V. Moody, S. Eswara Rao, T. Yokonuma, Toroidal Lie algebras and vertex representations, Geom.Ded., 35 (1990), 283-307. 